\title{
Experimental measurement of binding energy, selectivity and allostery using fluctuation theorems
}

\author{
J. Camunas-Soler, ${ }^{1,2,3}$ A. Alemany, ${ }^{1,2,4}$ and F. Ritort ${ }^{1,2}$ \\ ${ }^{1}$ Small Biosystems Lab, Departament de Física de la Matèria Condensada, \\ Facultat de Física, Universitat de Barcelona, Barcelona, Spain \\ ${ }^{2}$ CIBER de Bioingeniería, Biomateriales y Nanomedicina, \\ Instituto de Salud Carlos III, Madrid, Spain \\ ${ }^{3}$ Present address: Departments of Bioengineering and Applied Physics, \\ Stanford University, Stanford, California 94304, USA \\ ${ }^{4}$ Present address: Hubrecht Institute-KNAW and University \\ Medical Center Utrecht, 3584 CT Utrecht, the Netherlands
}

\begin{abstract}
Thermodynamic bulk measurements of binding reactions rely on the validity of the law of mass action and the assumption of a dilute solution. Yet important biological systems such as allosteric ligand-receptor binding, macromolecular crowding, or misfolded molecules may not follow these assumptions and require a particular reaction model. Here we introduce a fluctuation theorem for ligand binding and an experimental approach using single-molecule force-spectroscopy to determine binding energies, selectivity and allostery of nucleic acids and peptides in a model-independent fashion. A similar approach could be used for proteins. This work extends the use of fluctuation theorems beyond unimolecular folding reactions, bridging the thermodynamics of small systems and the basic laws of chemical equilibrium.
\end{abstract}


Binding energies are key quantities determining the fate of intermolecular reactions [1]. Bulk experimental approaches such as surface plasmon resonance, isothermal titration calorimetry and fluorescent ligand binding assays, allow the extraction of binding energies $\left(\Delta G_{\text {bind }}^{0}\right)$ from measurements of the dissociation constant $\left(K_{d}\right)$ with accuracy $\sim 1 \mathrm{kcal} / \mathrm{mol}$ through the expression:

$$
\Delta G_{\text {bind }}^{0}=-k_{\mathrm{B}} T \log \left[K_{d}\right],
$$

where $k_{\mathrm{B}}$ is the Boltzmann constant, and $T$ the temperature [2, 3]. However, many ligands such as DNA-binding proteins display different binding modes with varying affinities, or require the concerted action of several subunits, making quantitative measurements challenging [4, 5].

Force techniques such as optical tweezers can be used to pull on individual ligand-DNA complexes allowing detection of binding events one-at-a-time (Figure 1a, inset) [6-11]. However, force-induced ligand unbinding usually takes place in non-equilibrium conditions, and binding energies cannot be directly inferred from the measured work values. The Crooks fluctuation theorem and the Jarzynski equality [12, 13] are tools to extract equilibrium free energy differences from work distributions obtained far from equilibrium, allowing the measurement of folding free energies of nucleic acids and proteins, both from fully equilibrated [14 16] and kinetic states [17-19]. However, to date the use of fluctuation theorems remains restricted to unimolecular reactions (e.g. folding).

Here we introduce a fluctuation theorem for ligand binding (FTLB) that allows us to directly extract binding energies of bimolecular or higher-order reactions from irreversible work measurements in pulling experiments (see S1.1 in [20]). We first show how cyclic protocols allow an unambiguous classification of experimental pathways in relation to the initial and final state, which is an essential step in the application of these theorems. We then apply the FTLB to directly verify the validity of the law of mass action for dilute ligand solutions. Next we use the FTLB to accurately measure specific and nonspecific binding energies, as well as allosteric effects due to the cooperative binding of ligand pairs. Finally, we show how the FTLB is also applicable to extract binding energies to non-native structures (e.g. misfolded states, prions, chaperones), a measurement inaccesible to most bulk techniques [21 23].

As a proof of principle we investigated the binding of the restriction endonuclease EcoRI to a 30-bp DNA hairpin that contains its recognition site (GAATTC) (see S1.2,S1.3 in 
[20]). Restriction endonucleases, which bind their cognate sequences with high affinity, are a paradigm of protein-DNA interactions [24, 25]. In a typical experiment, the hairpin is unfolded (refolded) by increasing (decreasing) the distance $(\lambda)$ between the optical trap and the micropipette (Figure 1a). In the absence of ligand, the hairpin folds and unfolds in the force range $F_{c} \sim 12-15 \mathrm{pN}$. The binding of EcoRI increases the stability of the hairpin leading to higher unfolding forces $(\sim 23 \mathrm{pN})$. During a pulling experiment, EcoRI binds DNA when the hairpin is folded. However, since there is no net change in molecular extension upon binding/unbinding, the native $(N)$ and bound $(B)$ states cannot be distinguished at low forces. In contrast, at forces above $F_{c} \sim 12-15 \mathrm{pN}$ the bound state $(B)$ can be unambiguously distinguished from the unfolded state $(U)$, as the hairpin remains folded when the protein is bound but unfolds when it is unbound (Figure 1a, empty, blue and cyan dots respectively).

The FTLB is based on the extended fluctuation relation [17-19], and relates the work $(W)$ performed along a pulling protocol connecting the different EcoRI-hairpin binding states $(N, B, U)$ to their thermodynamic free-energy differences. We performed cyclic protocols that start and end at a force $\sim 21 \mathrm{pN}$ (Figure $1 \mathbf{b}$ inset and Fig. S1). This force is well above $F_{c} \sim 12-15 \mathrm{pN}$, resulting in paths that connect states $U$ and $B$, that are then classified into four different sets according to their initial and final states $(U \rightarrow U, U \rightarrow B$, $B \rightarrow U, B \rightarrow B$, Figure 1c). We repeatedly pulled the hairpin and measured the partial work distributions and fraction of paths connecting states $U$ and $B\left(P^{B \rightarrow U}(W), P^{U \rightarrow B}(W)\right.$, $\phi^{B \rightarrow U}, \phi^{U \rightarrow B}$ respectively) and extracted the free-energy difference between states $B$ and $U$, $\Delta G_{B U}\left(=G_{U}-G_{B}\right)$ using the FTLB (see S1.4 in [20]):

$$
\frac{\phi^{B \rightarrow U}}{\phi^{U \rightarrow B}} \frac{P^{B \rightarrow U}(W)}{P^{U \rightarrow B}(-W)}=\exp \left[\frac{W-\Delta G_{B U}}{k_{\mathrm{B}} T}\right] .
$$

We performed experiments at different EcoRI concentrations, and determined $\Delta G_{B U}$ from the work value $(\widetilde{W})$ at which the partial work distributions cross $\left(P^{B \rightarrow U}(\widetilde{W})=P^{U \rightarrow B}(-\widetilde{W})\right)$ by taking $\Delta G_{B U}=\widetilde{W}+k_{\mathrm{B}} T \log \left(\phi^{U \rightarrow B} / \phi^{B \rightarrow U}\right)$ (Figure 1d, S1.5 in [20]). The term $\Delta G_{B U}$ includes all the energetic contributions involved in going from $B$ to $U$ (e.g. binding energy, conformational changes, elastic terms, see S1.6 in [20]). By subtracting the elastic contributions and the energy of formation of the hairpin from the measured $\Delta G_{B U}$ value, we extract the binding energy at zero force $\left(\Delta G_{\text {bind }}\right)$ at different EcoRI concentrations (Figure 1e and Tables S1, S2). As shown in Figure 1e, $\Delta G_{\text {bind }}$ follows the law of mass action (Eq. 1), 
$\Delta G_{\text {bind }}=\Delta G_{\text {bind }}^{0}+k_{\mathrm{B}} T \log \left(C / C_{0}\right)$ with $\Delta G_{\text {bind }}^{0}=26 \pm 0.5 k_{\mathrm{B}} T$, providing a direct test of its validity. This value is independent on the start/end force of the cyclic protocol and relies on a correct classification of paths (Fig. S2-S3). We also performed titration experiments with varying $\mathrm{NaCl}$ concentration showing that EcoRI binding energy has a pronounced saltdependency with slope $m_{[\mathrm{NaCl}]}=-11 \pm 2 k_{\mathrm{B}} T$ (Figure 1 f and Tables S3, S4), in agreement with previous bulk experiments [26, 27]. Finally, we repeated experiments with hairpins containing non-cognate DNA sequences which did not show binding in the same range of EcoRI concentrations, proving the specificity of the interaction [6, 9].

To further test the validity of Eq. 2, we investigated a model system consisting of a short oligonucleotide of 10 bases that binds a DNA hairpin. The oligonucleotide can bind the substrate by base-pairing complementarity when the hairpin is in the unfolded $(U)$ state, thereby inhibiting the refolding of the hairpin at low forces. At forces below the critical force range of the hairpin $\left(F_{c} \sim 8-10 \mathrm{pN}\right)$, the oligo-bound state $(B)$ competes with the formation of the native hairpin $(N)$, and states $B$ and $N$ can be distinguished due to their different molecular extension (Figure 2a). To apply Eq. 2, we considered cyclic protocols that start and end at a force lower than the range $F_{c}$ (Figure $2 \mathbf{b}$ ). From the measured partial work distributions and fractions of paths connecting $N$ and $B$ (Figure 2c) we extracted the binding energies at zero force $\left(\Delta G_{\text {bind }}\right)$ (Figure $2 \mathbf{d}$ and Tables S5, S6). Measured binding energies again follow the law of mass action with $\Delta G_{\text {bind }}^{0}=22 \pm 1 k_{\mathrm{B}} T$ (Figure 2d). This agrees with theoretical predictions using the nearest-neighbour model $\left(\Delta G_{\mathrm{th}}^{0}=22 k_{\mathrm{B}} T\right)[28,29]$ and equilibrium experiments performed at the coexistence force of the hairpin, where hopping due to binding/unbinding is observed (Figs. S4-S6 and Table S7). The inclusion of the ratio $\phi^{N \rightarrow B} / \phi^{B \rightarrow N}$ (Figure 2d, inset) is essential to recover the correct binding energies. Previous attempts to derive binding energies using unidirectional work measurements and the Jarzynski equality did not account for concentration-dependent effects in the chemical potential that are essential in Equation 2 [9] .

To prove the general power of the method, we studied echinomycin, a small DNA bisintercalator with selectivity for CG steps [30] that binds contiguous ACGT sites cooperatively [31. We performed experiments with a 12-bp DNA hairpin containing a single CG-step (SP hairpin) that shows rupture forces in the range $F_{c} \sim 6-8 \mathrm{pN}$ (Figure $3 \mathbf{a}$ and Fig. S7). In the presence of echinomycin the histogram of rupture forces is shifted to higher values and shows a bimodal distribution, indicating two binding modes: a high-affinity binding to the 
specific CG-site (high-force peak, $\sim 18 \mathrm{pN}$ ), and a low-affinity binding to other non-specific sites (low-force peak, $\sim 12 \mathrm{pN}$ ). To confirm this, we pulled a hairpin in which we removed the specific binding site by inverting the CG-motif (NSP hairpin). In the presence of ligand only the low affinity peak is observed (Figure 3a).

To extract the binding energy of each mode, we performed cyclic protocols that start at a force high enough to discriminate both binding modes: we used $\sim 18 \mathrm{pN}(\sim 13 \mathrm{pN})$ for the SP (NSP) hairpin in order to extract both the specific and nonspecific binding energy of the ligand. In this way, we obtained paths connecting states $B$ and $U$, and extracted the binding energy of the specific and nonspecific modes (Tables S8-S11). For both binding modes, $\Delta G_{\text {bind }}$ follows the law of mass action with $\Delta G_{\text {bind,SP }}^{0}=20.0 \pm 0.8$ $k_{\mathrm{B}} T$ and $\Delta G_{\text {bind,NSP }}^{0}=13.2 \pm 0.5 k_{\mathrm{B}} T$ (Figure $3 \mathbf{b}$ ), which give affinities of $2 \mathrm{nM}$ and 1.8 $\mu \mathrm{M}$ respectively (Eq. 1). This measurement of an affinity in the $\mathrm{nM}$ range for the specific binding is compatible with quasi-equilibrium experiments (Fig. S8) and improves previous studies where accurate measurements could not be obtained due to the concurrent action of both modes [32].

The FTLB allows us to go beyond free-energy measurements of single ligands, and measure allosteric effects between ligands binding at nearby positions [5]. For this, we designed hairpin $N C$ which contains two ACGT sites separated by 2 bp (Figure 3c). The simultaneous binding of two ligands can be distinguished from the binding of a single ligand from the force rips observed in the force-distance curve (Fig. S9). By applying the FTLB we extracted the binding energy per ligand in the single and double bound states, and found that binding is favoured by the presence of a neighbouring ligand. The FTLB allows us to quantitatively test the distance-dependence of this allosteric effect by performing a differential measurement of binding energies with hairpin $C$, which contains two contiguous sites (Figure 3c, Tables S12-S14). The binding energy per ligand we obtain in the double bound state in hairpin $C$ is $2.4 \pm 0.5 k_{\mathrm{B}} T$ higher than in hairpin $N C$, providing a direct experimental measurement of cooperativity effects in ligand pairs as a function of their distance.

Single-molecule manipulation is particularly suited to observe the formation of misfolded structures (e.g. prions, amyloids) [23, 33], but methods to characterize binding to these species are currently lacking. By applying the FTLB it is possible to extract the binding energy to these kinetically stabilized non-native structures. By using a DNA hairpin with two binding sites separated by $4 \mathrm{bp}$, we observe the formation of a misfolded structure consisting 
of two short (4bp) hairpins in series (Figure 3d, hairpin M). Such an off-pathway kinetic state is unobservable in the absence of ligand due to its low energy of formation, however it is kinetically stabilized by the binding of the ligand. We applied Eq. 2 by choosing a starting point of the cyclic protocol where the native-bound and misfolded-bound conformations are distinguishable $(F \sim 10 \mathrm{pN})$, and found that the energy of binding to both configurations are equal $\left(\Delta G_{\text {bind,M-N }}=2 \pm 1 k_{\mathrm{B}} T\right.$, Fig. S10 and Tab. S15, S16).

In this work, we have introduced a fluctuation theorem for ligand binding (FTLB) to directly determine binding energies as a function of ligand concentration in single-molecule experiments. Using different biomolecular systems of increasing complexity we provide a single-molecule verification of the law of mass action, and show how the FTLB can account for mass exchange between a molecular system and the environment. We can resolve binding energies to specific and non-specific sites with affinities spanning six orders of magnitude. The FTLB provides a direct experimental measurement of binding energies without assuming any model or reaction scheme, which is particularly useful in cases where the law of mass action does not hold. To show this, we applied the FTLB in two situations where this may happen: the cooperative binding of multiple ligands to the same substrate and the stabilization of kinetic structures through ligand binding - both measurements inaccessible to bulk methods and relevant to many interactions between proteins and ligands.

The use of an inherently non-equilibrium method to obtain equilibrium binding energies also grants access to molecular interactions that equilibrate over very long timescales (e.g. nucleosome assembly) and that can only be currently measured by indirect techniques such as competition assays [2, 22, 34]. The FTLB relates work measurements to binding energies without making any assumption on reaction kinetics or the ideal solution limit. Therefore it might be also used to test the explicit breakdown of the law of mass action in conditions where it is not applicable, for instance in crowded environments, where ligands exhibit compartmentalized dynamics due to steric hindrance interactions [35]. Lastly, the applicabilty of the FTLB is not restricted to biomolecular reactions, and might be directly applied to other interacting systems that can only be explored through non-equilibrium methods. 


\section{ACKNOWLEDGEMENTS}

All authors acknowledge funding from grants ERC MagReps 267 862, FP7 grant Infernos 308850, Icrea Academia 2013 and FIS2013-47796- P.

All data used in this study are included in the main text and in the supplementary materials.

\section{AUTHOR CONTRIBUTIONS}

J.C.-S. and A.A. equally contributed to this work. 


\section{FIGURE CAPTIONS}

\section{Figure 1}
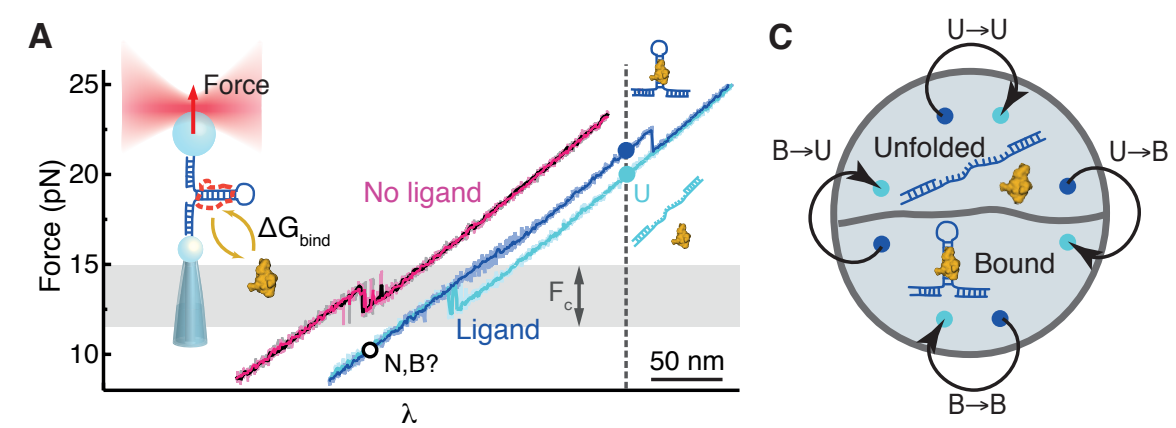

B
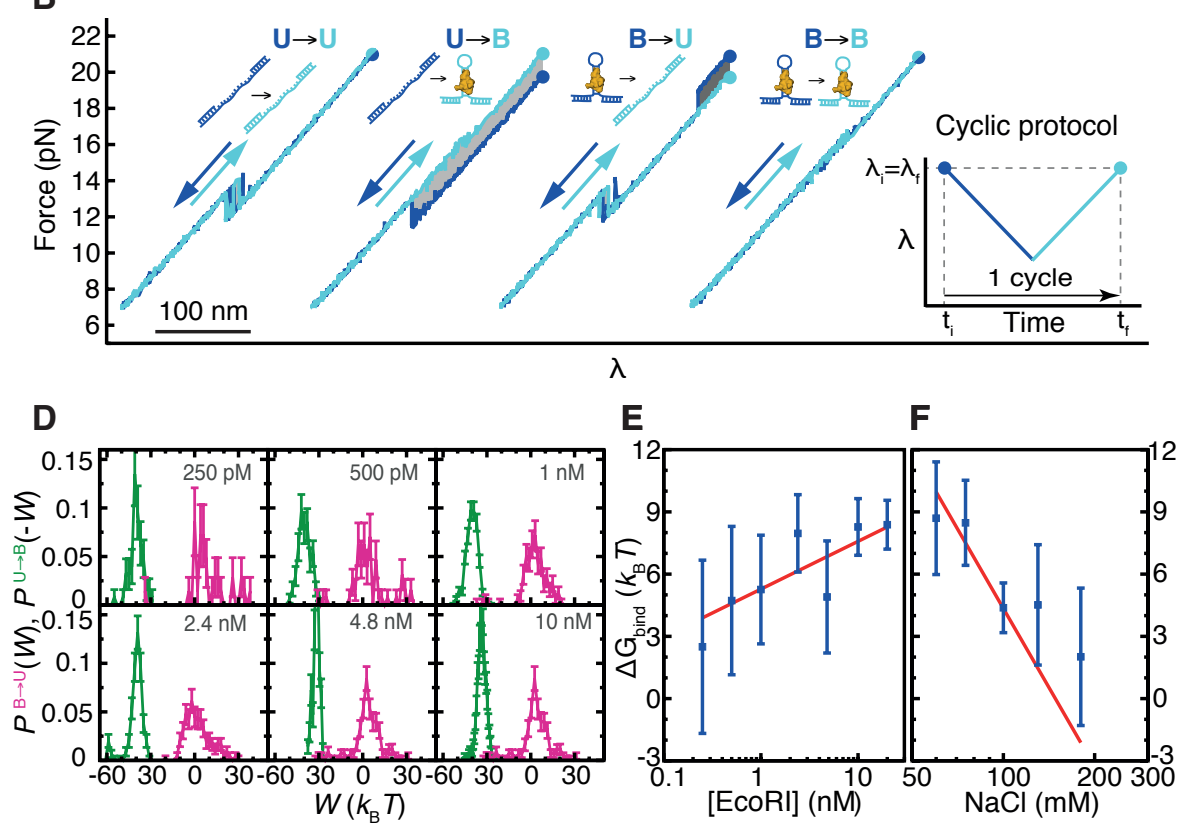

EcoRI binding to DNA. (a) Unfolding/refolding force-distance curves of a DNA hairpin in the absence (magenta/black) and presence (blue/cyan) of EcoRI protein. The bound $(B)$ and unfolded $(U)$ states are discriminated at high force by the presence of two distinct force branches. (b) Cyclic pulling curves classified according to their initial (blue dot) and final state (cyan dot) that start and end at a high force $(\sim 21 \mathrm{pN})$. Work equals the enclosed area between the two curves and is shown in dark/light gray for positive/negative values. (c) Paths of a non-equilibrium cyclic protocol connecting different initial and final states. (d) Partial work distributions of $U \rightarrow B$ (green) and $B \rightarrow U$ (magenta) transitions at different EcoRI concentrations. (e) Binding energy of EcoRI (blue) and fit to the law of mass action (red line) at $\left(130 \mathrm{mM} \mathrm{Na}^{+}, 25^{\circ} \mathrm{C}, C_{0}=1 \mathrm{M}\right)$. (f) Binding energy of EcoRI at varying 
[NaCl] (1 nM EcoRI). Error bars were obtained from bootstrap using 1000 re-samplings of size N ( $\mathrm{N}$ is total number of pulls for each condition shown in Tables S1 and S3). 


\section{Figure 2}
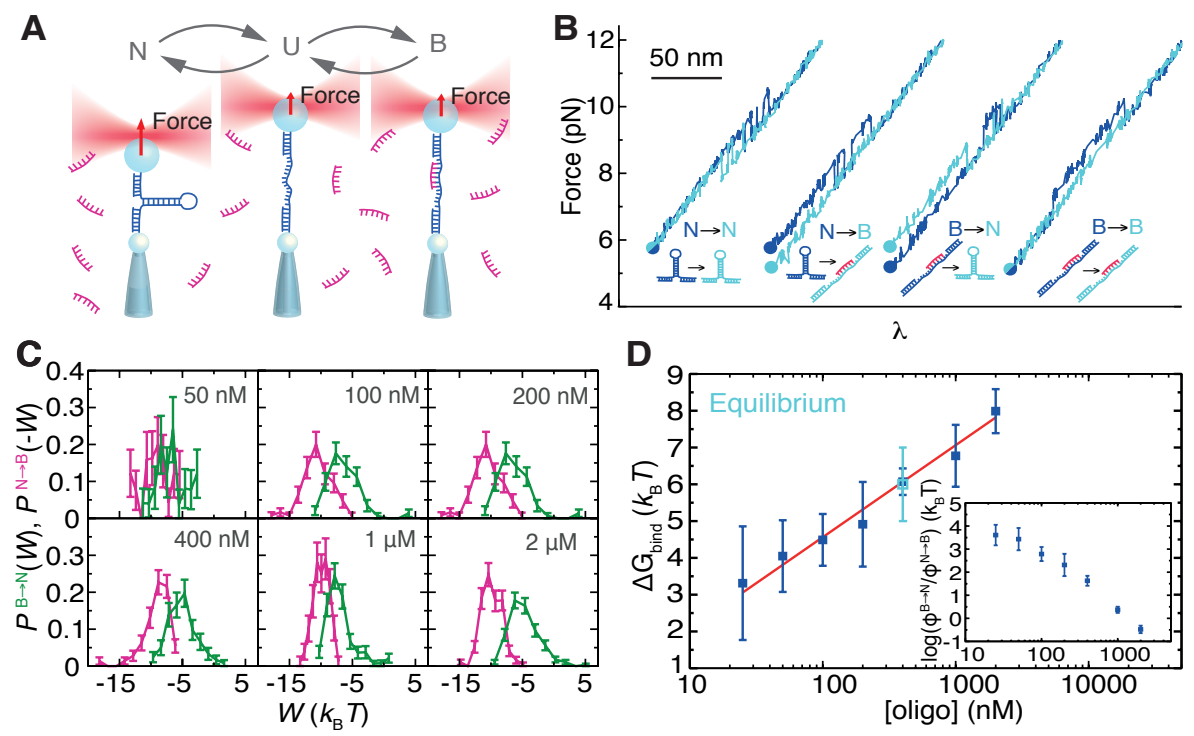

Oligo binding to DNA. (a) Scheme of native $(N)$, unfolded $(U)$ and oligo bound $(B)$ states. (b) Cyclic pulling curves that start and end at low forces ( $\sim 6 \mathrm{pN})$ classified according to their initial (blue dot) and final state (cyan dot). (c) Partial work distributions of $B \rightarrow N$ (green) and $N \rightarrow B$ (magenta) transitions. (d) Binding energy of the 10-base oligo (blue) and fit to the law of mass action (red line). The value obtained from hopping equilibrium experiments at [oligo] $=400 \mathrm{nM}$ (see S1.7 in [20]) is shown in cyan. (Inset) Contribution of the ratio $\phi^{N \rightarrow B} / \phi^{B \rightarrow N}$ to the binding energy. Error bars were obtained from bootstrap as described in Fig. 1. 


\section{Figure 3}
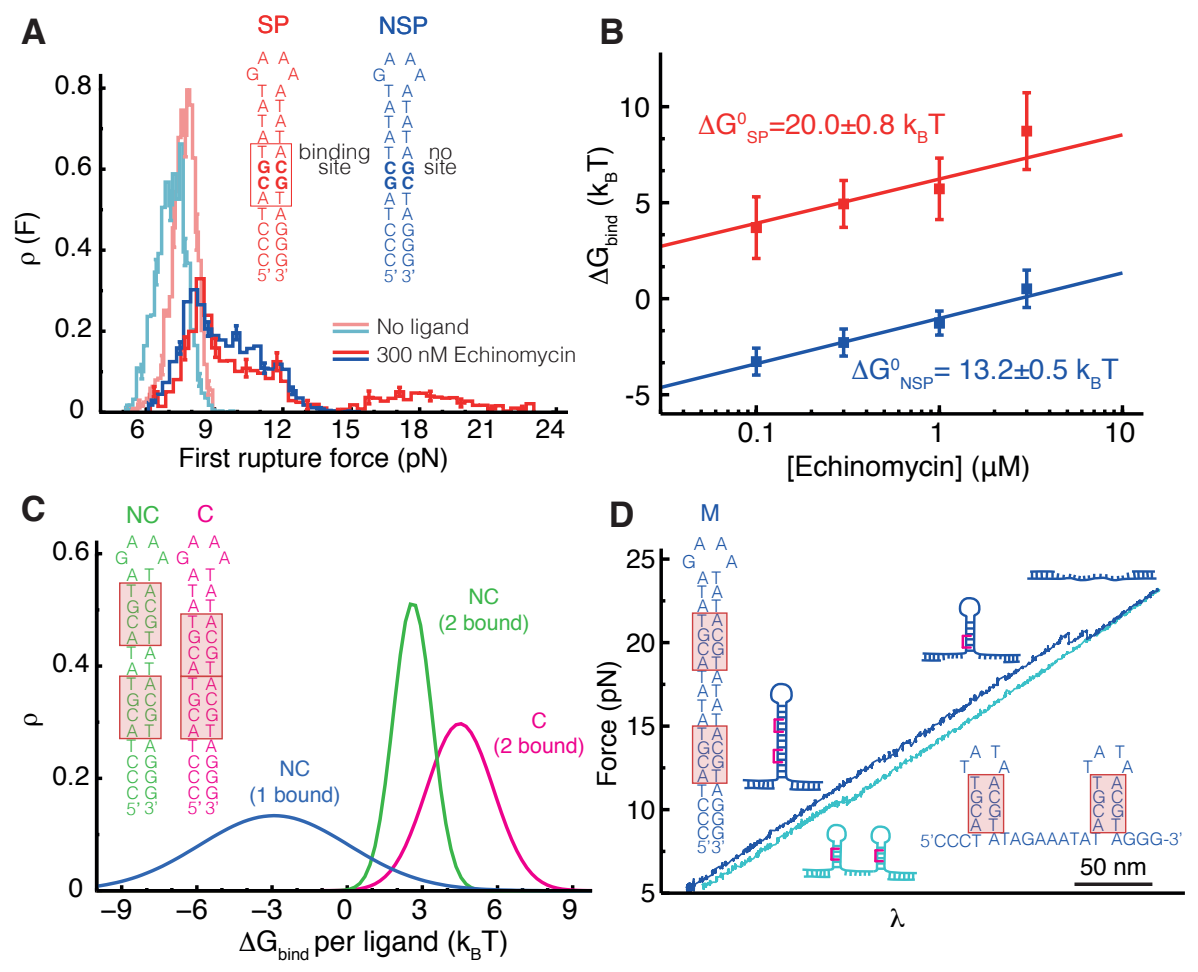

Binding specificity, allostery and kinetic stabilization of misfolded states for the peptide Echinomycin. (a) First rupture force distribution of hairpins SP (red) and NSP (blue) in the absence (light) and presence (dark) of Echinomycin. (b) Binding energy of Echinomycin to a specific (red) and nonspecific (blue) site, and fit to the law of mass action. (c) Hairpins $\mathrm{C}$ and $\mathrm{NC}$ contain two specific binding sites (red boxes) placed contiguously or separated by 2-bp respectively. Binding energy per ligand when one (blue) or two ligands (green) are bound to hairpins $\mathrm{NC}$ or $\mathrm{C}$ (magenta) ([Echninomycin] $]=3 \mu \mathrm{M})$. Gaussian distributions are reconstructed from mean and variance of measurements. The wider distribution for the single bound state in hairpin NC (blue) is due to the lower number of paths reaching this state at high ligand concentration, increasing measurement error. (d) Pulling cycle of hairpin M in the presence of Echinomycin. The unfolding curve (blue) shows two force rips at $F \sim 20 \mathrm{pN}$ corresponding to the unbinding of two ligands bound to specific sites. In the refolding curve (cyan), the hairpin does not fold back to the native state, and misfolds into a kinetically stabilized configuration of longer molecular extension $(\sim 40 \%$ of refolding curves at $[$ Echninomycin $]=10 \mu \mathrm{M})$. Error bars were obtained from bootstrap as described in Fig. 1. 


\section{MATERIALS AND METHODS}

\section{Mathematical proof of the Fluctuation Theorem for Ligand Binding (FTLB)}

The FTLB is derived following the same steps as in [18]. Consider a system with a fluctuating number of particles $N$, which correspond to the ligand molecules. The system evolves under an experimental protocol $\lambda(t)$, where $\lambda$ denotes the control parameter and in our case corresponds to the position of the optical trap relative to the pipette. We discretize in time the protocol as $\lambda(t)=\left\{\lambda_{0}, \lambda_{1}, \ldots, \lambda_{t_{f}}\right\}$, where $\lambda_{i}\left(i=0,1, \ldots, t_{f}\right)$ denotes the value of $\lambda$ at the time of the protocol $t=i \Delta t$ (being $\Delta t$ the time discretization unit), and $t_{f}$ denotes the duration of the protocol. Along the protocol $\lambda(t)$ the system follows a given trajectory $\Gamma$, where a sequence of configurations $\mathcal{C}$ are sampled. The trajectory can be discretized as $\Gamma=\left\{\mathcal{C}_{0}, \mathcal{C}_{1}, \ldots, \mathcal{C}_{t_{f}}\right\}$. Each configuration $\mathcal{C}_{i}\left(i \in 0,1, \ldots, t_{f}\right)$ is characterized by the number of particles, $N_{i}$, and the degrees of freedom of each particle.

The equilibrium probability to be in a given configuration $\mathcal{C}_{i}$ at $\lambda$ can be written, according to the grand-canonical ensemble, as:

$$
P^{\mathrm{eq}}\left(\mathcal{C}_{i}\right)=\frac{z^{N_{i}} e^{-\beta E_{\lambda}\left(\mathcal{C}_{i}\right)}}{Z^{G C}}, \quad \quad Z^{G C}=\sum_{N_{i}} \sum_{\mathcal{C}_{i}\left(N_{i}\right)} z^{N_{i}} e^{-\beta E_{\lambda}\left(\mathcal{C}_{i}\right)}
$$

where $\beta=\left(k_{\mathrm{B}} T\right)^{-1}$ (being $k_{\mathrm{B}}$ the Boltzmann constant and $T$ the absolute temperature), $z$ is the fugacity of the system (equal to $e^{\beta \mu}$, being $\mu$ the chemical potential of the ligand molecules), $Z^{G C}$ is the grand canonical partition function, and $E_{\lambda}\left(\mathcal{C}_{i}\right)$ is the energy of the configuration $\mathcal{C}_{i}$ at $\lambda$.

We suppose that the dynamics of the system satisfy the following detailed balance condition:

$$
\frac{P\left(\mathcal{C}_{t} \rightarrow \mathcal{C}_{t+1}\right)}{P\left(\mathcal{C}_{t+1} \rightarrow \mathcal{C}_{t}\right)}=z^{N_{t+1}-N_{t}} e^{-\beta\left(E_{\lambda(t+1)}\left(\mathcal{C}_{t+1}\right)-E_{\lambda(t+1)}\left(\mathcal{C}_{t}\right)\right)}
$$

Therefore, the probability of the system to follow a given trajectory $\Gamma$ (without imposing any initial and final configuration), and the probability to follow its time reversed $\hat{\Gamma}$ is:

$$
P(\Gamma)=\prod_{t=0}^{t_{f}-1} P\left(\mathcal{C}_{t} \rightarrow \mathcal{C}_{t+1}\right), \quad \hat{P}(\hat{\Gamma})=\prod_{t=0}^{t_{f}-1} P\left(\hat{\mathcal{C}}_{t} \rightarrow \hat{\mathcal{C}}_{t+1}\right)
$$

where $\hat{\mathcal{C}}_{t}=\mathcal{C}_{t_{f}-t}$.

We assume that in the forward protocol $\lambda(t)$ the system starts in partial equilibrium at $\mathcal{C}_{0}$, while in the reversed protocol $\hat{\lambda}(t)$ it starts in partial equilibrium at $\hat{\mathcal{C}}_{0}=\mathcal{C}_{t_{f}}$ (and 
$\left.\hat{\lambda}(0)=\lambda\left(t_{f}\right)\right)$. The partial equilibrium probability density function of a given configuration $\mathcal{C}_{i} \in S$, where $S$ is a subset of configurations accessible by the system, can be written as [18]:

$$
P_{S}^{\mathrm{eq}}\left(\mathcal{C}_{i}\right)=\chi_{S}\left(\mathcal{C}_{i}\right) \frac{z^{N_{i}} e^{-\beta E\left(\mathcal{C}_{i}\right)}}{\sum_{N_{i}} \sum_{\mathcal{C}_{i} \in S} z^{N_{i}} e^{-\beta E\left(\mathcal{C}_{i}\right)}}=\chi_{S}\left(\mathcal{C}_{i}\right) P^{\mathrm{eq}}\left(\mathcal{C}_{i}\right) \frac{Z^{G C}}{Z_{S}^{G C}},
$$

where $\chi_{S}\left(\mathcal{C}_{i}\right)$ is equal to one if $\mathcal{C}_{i} \in S$ and zero otherwise, and $Z_{S}^{G C}$ is the grand canonical partition function restricted to the subset $S$.

Suppose that the system starts in non-equilibrium conditions. Particularly, the system starts in partial equilibrium at the kinetic state $S_{0}$ in the forward trajectory and at at the kinetic state $S_{t_{f}}$ in the reversed one. A kinetic state is a partially equilibrated region of configurational space, meaning that during a finite amount of time the system is confined and thermalized within that region. This is mathematically described by a Boltzmann-Gibbs distribution restricted to configurations contained in that region. Then:

$$
\begin{aligned}
\frac{P_{\lambda(0), S_{0}}^{\mathrm{eq}}\left(\mathcal{C}_{0}\right) P(\Gamma)}{P_{\lambda\left(t_{f}\right), S_{t_{f}}}^{\mathrm{eq}}\left(\mathcal{C}_{t_{f}}\right) \hat{P}(\hat{\Gamma})} & =\frac{\chi_{S_{0}}\left(\mathcal{C}_{0}\right) P_{\lambda(0)}^{\mathrm{eq}}\left(\mathcal{C}_{0}\right) Z_{\lambda(0)}^{G C}}{Z_{\lambda(0), S_{0}}^{G C}} \frac{Z_{\lambda\left(t_{f}\right), S_{t_{f}}}^{G C}}{\chi_{S_{t_{f}}}\left(\mathcal{C}_{t_{f}}\right) P_{\lambda\left(t_{f}\right)}^{\mathrm{eq}}\left(\mathcal{C}_{t_{f}}\right) Z_{\lambda\left(t_{f}\right)}^{G C}} \frac{P(\Gamma)}{\hat{P}(\hat{\Gamma})} \\
& =\frac{\chi_{S_{0}}\left(\mathcal{C}_{0}\right) Z_{\lambda\left(t_{f}\right), S_{t_{f}}}^{G C}}{\chi_{S_{t_{f}}}\left(\mathcal{C}_{t_{f}}\right) Z_{\lambda(0), S_{0}}^{G C}} e^{\beta W(\Gamma)},
\end{aligned}
$$

where:

$$
W(\Gamma)=\sum_{t=0}^{t_{f}-1}\left(E_{\lambda(t+1)}\left(\mathcal{C}_{t}\right)-E_{\lambda(t)}\left(\mathcal{C}_{t}\right)\right),
$$

is as the work exerted upon the system along the forward process.

Now we compute the average of the quantity $\mathcal{O} e^{-\beta W}$ over the forward trajectories that start in partial equilibrium in a configuration $\mathcal{C}_{0} \in S_{0}$ and end in $\mathcal{C}_{t_{f}} \in S_{t_{f}}$. Therefore:

$$
\begin{aligned}
& \left\langle\mathcal{O} e^{-\beta W}\right\rangle^{S_{0} \rightarrow S_{t_{f}}}=\frac{\sum_{\Gamma} P_{\lambda(0), S_{0}}^{\mathrm{eq}}\left(\mathcal{C}_{0}\right) P(\Gamma) \chi_{S_{t_{f}}}\left(\mathcal{C}_{t_{f}}\right) \mathcal{O}(\Gamma) e^{-\beta W(\Gamma)}}{\sum_{\Gamma} P_{\lambda(0), S_{0}}^{\mathrm{eq}}\left(\mathcal{C}_{0}\right) P(\Gamma) \chi_{S_{t_{f}}}\left(\mathcal{C}_{t_{f}}\right)} \\
& =\frac{\phi^{S_{t_{f}} \rightarrow S_{0}}}{\phi^{S_{0} \rightarrow S_{t_{f}}}} \frac{Z_{\lambda\left(t_{f}\right), S_{t_{f}}}^{G C}}{Z_{\lambda(0), S_{0}}^{G C}} \frac{\sum_{\Gamma} P_{\hat{\lambda}\left(t_{0}\right), \hat{S}_{0}}^{\mathrm{eq}}\left(\hat{\mathcal{C}}_{0}\right) \hat{P}(\hat{\Gamma}) \chi_{\hat{S}_{t_{f}}}\left(\hat{\mathcal{C}}_{t_{f}}\right) \hat{\mathcal{O}}(\hat{\Gamma})}{\sum_{\Gamma} P_{\hat{\lambda}\left(t_{0}\right), \hat{S}_{0}}^{\mathrm{eq}}\left(\hat{\mathcal{C}_{0}}\right) \hat{P}(\hat{\Gamma}) \chi_{\hat{S}_{t_{f}}}\left(\hat{\mathcal{C}}_{t_{f}}\right)} \\
& =\frac{\phi^{S_{t_{f}} \rightarrow S_{0}}}{\phi^{S_{0} \rightarrow S_{t_{f}}}} \frac{Z_{\lambda\left(t_{f}\right), S_{t_{f}}}^{G C}}{Z_{\lambda(0), S_{0}}^{G C}}\langle\hat{\mathcal{O}}\rangle^{S_{t_{f}} \rightarrow S_{0}} .
\end{aligned}
$$

By defining $\mathcal{O}(\Gamma)=\delta(W-W(\Gamma))$ we obtain the extended Crooks relation in the grandcanonical ensemble, or equivalently the Fluctuation Theorem for Ligand Binding (FTLB): 


$$
\begin{aligned}
\frac{\phi^{S_{0} \rightarrow S_{f}}}{\phi^{S_{t_{f}} \rightarrow S_{0}}} \frac{P^{S_{0} \rightarrow S_{t_{f}}}(W)}{P^{S_{t_{f}} \rightarrow S_{0}}(-W)} & =e^{\beta W} \frac{Z_{\lambda\left(t_{f}\right), S_{t_{f}}}^{G C}}{Z_{\lambda(0), S_{0}}^{G C}} \\
& =\exp \left[\beta\left(W-\Delta G_{S_{0} S_{t_{f}}}\right)\right]
\end{aligned}
$$

where $\Delta G_{S_{0} S_{t_{f}}}=G\left(\lambda\left(t_{f}\right), S_{t_{f}}\right)-G\left(\lambda(0), S_{0}\right)$.

\section{Force spectroscopy experiments with optical tweezers}

Experiments are performed with a highly stable miniaturized dual-beam optical tweezers described in previous studies [29]. The DNA hairpins are tethered between two beads by using short dsDNA handles (29-bp) that are differentially end-labelled with biotin and digoxigenin to attach each handle to a different bead (see Supp. Section 5 for hairpin sequences and synthesis details) [36]. Pulling speed is set at $190 \mathrm{~nm} / \mathrm{s}$ in all the experiments. For the EcoRI experiments, longer dsDNA handles ( 600-bp) are used to reduce nonspecific interactions mediated by the protein and beads. For these experiments, the microfluidics chamber was also coated with Poly(ethylene glycol) (PEG) to avoid protein loss due to nonspecific absorption on the glass surface [37]. Experimental conditions for each interaction were chosen to be comparable to previous ensemble and single-molecule studies: for

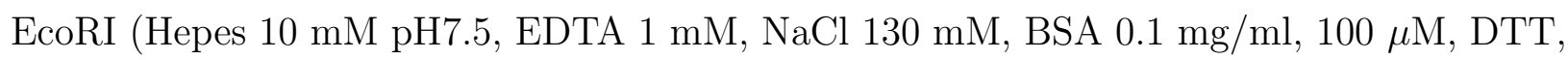
$0.01 \% \mathrm{NaN}_{3}$ ) and salt titrated in the range $60-180 \mathrm{mM} \mathrm{NaCl}$; for the oligonucleotide (Tris

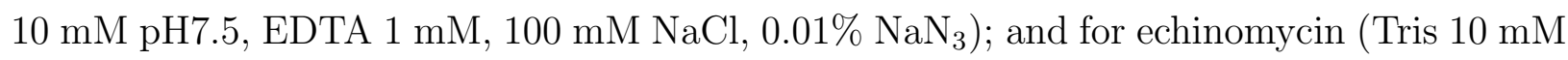
pH7.5, EDTA $1 \mathrm{mM}, 100 \mathrm{mM} \mathrm{NaCl}, 2 \%$ DMSO, 0.01\% $\mathrm{NaN}_{3}$ ). All ligands were obtained from commercial sources and used without further purification: EcoRI (New England Biolabs, $100 \mathrm{U} / \mu \mathrm{l}, \sim 800 \mathrm{nM}$ dimer), oligonucleotide (Eurofins MWG Operon, HyPur grade), Echinomycin (Merck Millipore). Concentrations were confirmed using a spectrophotometric analysis for the oligonucleotide (extinction coefficient $97400 \mathrm{M}^{-1} \mathrm{~cm}^{-1}$ at $\lambda=260 \mathrm{~nm}$ ) and echinomycin (extinction coefficient $11500 \mathrm{M}^{-1} \mathrm{~cm}^{-1}$ at $\lambda=325 \mathrm{~nm}$ ), whereas for EcoRI we performed an electrophoretic mobility shift assay using previously described protocols [38]. All experiments were performed at $25^{\circ} \mathrm{C}$. In all the cases, the number of cycles obtained per molecule range between 20 and 1300. A minimum of 2 and a maximum of 10 molecules were pulled in each case (see tables I, III, V, VIII, X, XII, XV). 


\section{DNA hairpins used in this work}

DNA hairpin sequences, secondary structure, binding sites, thermodynamic information and synthesis details:

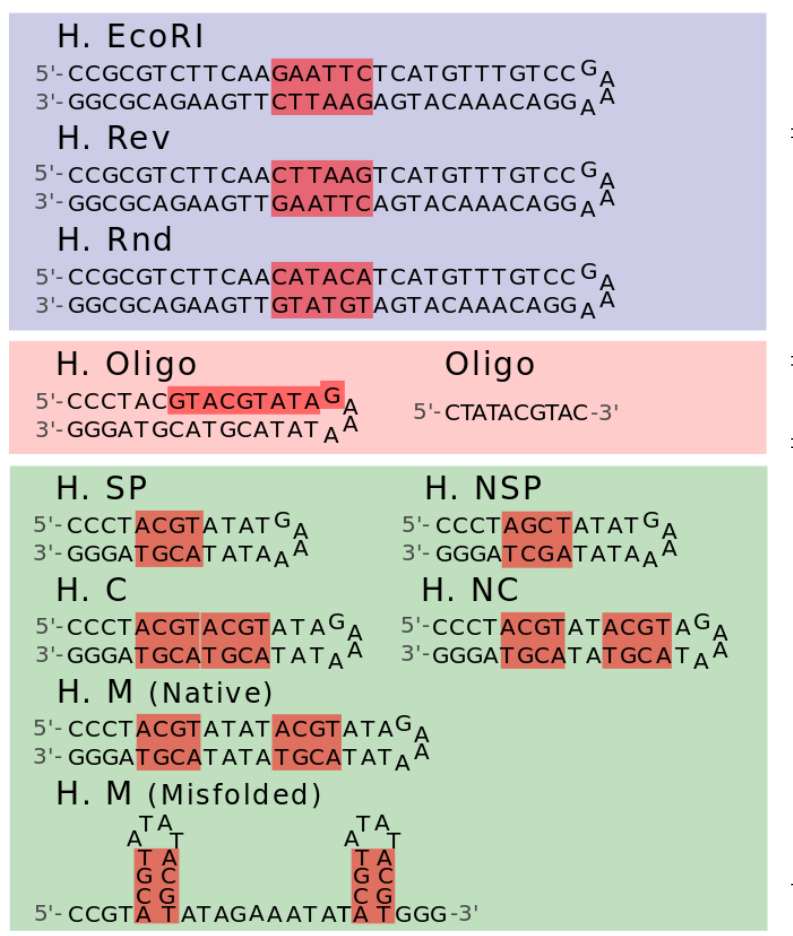

\begin{tabular}{lcc} 
Haipin & $\Delta G_{N U}^{0}\left(k_{\mathrm{B}} T\right)$ Handle length \\
\hline \hline EcoRI & $65 \pm 3$ & long \\
Rev & $67 \pm 2$ & long \\
Rnd & $68 \pm 2$ & long \\
\hline \hline Oligo & $28 \pm 1$ & short \\
\hline \hline SP & $19.7 \pm 0.5$ & short \\
NSP & $19.4 \pm 0.3$ & short \\
C & $18 \pm 1$ & short \\
NC & $18 \pm 1$ & short \\
M (Native $)$ & $33 \pm 1$ & short \\
\multicolumn{1}{c}{$($ Misfolded $)$} & $10 \pm 1$ & \\
\hline
\end{tabular}

Blue-Top. Hairpins used to study the binding of EcoRI to its recognition sequence (5'GAATTC-3'). Hairpin EcoRI contains the specific binding site, indicated in red; Hairpin Rev contains its reversed sequence (red); and Hairpin Rnd contains a random sequence (red). We did not observe binding of EcoRI to hairpins Rnd and Rev in pulling experiments using these hairpins.

Red-Middle. Hairpin (left) used to study the specific binding of an oligonucleotide (right) to its complementary ssDNA sequence. The binding site of the oligo is indicated in red.

Green-Bottom. Hairpins used to study the binding of echinomycin to different binding motifs. Hairpin SP contains a specific binding site 5'-ACGT-3' (red). In hairpin NSP the specific binding site is removed by doing a sequence permutation (red). Hairpin $\mathrm{C}$ contains two contiguous binding sites (red), while hairpin NC contains two binding sites separated by two basepairs (red). Hairpin M contains two binding sites that are separated by four base pairs (hairpin M, native). In the presence of echinomycin, a misfolded structure containing two serially connected hairpins (hairpin M, misfolded) becomes kinetically stabilized by the binding of the ligand to two 4-bp hairpins containing the 5'-ACGT-3' motif (red). 
Mean values for the free energy of formation at zero force at $25^{\circ} \mathrm{C}$ and $130 \mathrm{mM} \mathrm{NaCl}$ (which are the experimental conditions unless stated otherwise) have been obtained by averaging over the results provided by the nearest-neighbor model and the unified oligonucleotide set of basepair free energies measured in bulk [28, 39] and unzipping [29] experiments. Error bars are standard errors obtained between the two different estimations. To pull on the hairpins, handles of two different length are used in the experiments: short (29 bp) and long (500 bp) dsDNA handles.

For the experiments with echinomycin and the oligonucleotide we used a short handle construct (total handle length: 58-bp). This short handles construct is better suited to study small ligands that might non-specifically bind to the dsDNA handles. Due to the short length of this construct, it can be synthesized by direct annealing and ligation of three partially complementary oligonucleotides that create the hairpin structure and dsDNA handles, as described in previous studies [36].

For the experiments performed with EcoRI we used a long handle construct to maintain a larger separation between the two beads at low forces (total handle length: 1322-bp). The synthesis is similar to the protocol described in [36]. Briefly, the two handles are performed by PCR amplification of plasmid pBR322 to obtain DNA fragments that contain a restriction site for TspRI or Tsp45I respectively, but do not contain potential binding sites for the ligand (i.e. EcoRI). A biotin tag is introduced in one of the handles using a 5'-biotinylated primer on the PCR reaction. The other handle is tailed with digoxigenin-dUTP using the 3'-5' exonuclease activity of T4 DNA polymerase. After digestion of the labelled products with each enzyme, the TspRI/Tsp45I cohesive ends are used to anneal and ligate the handles to the the hairpin structure, that is assembled using oligonucleotides.

\section{Application of the FTLB: how to}

To apply the FTLB to cyclic pulling protocols we follow the next steps:

1. Identification of initial and final states in the non-equilibrium protocol.

In the particular cases studied in our work, initial and final states in the cyclic pulling experiments are:

- Specific binding of EcoRI to dsDNA: $B$ and $U$ (high forces). 
- Specific binding of an oligo to ssDNA: $B$ and $N$ (low forces).

- Specific binding of echinomycin to dsDNA: $B$ and $U$ (high forces).

- Non-specific binding of echinomycin to dsDNA: $B$ and $U$ (high forces).

- Cooperative binding of echinomycin to dsDNA: $B_{2}, B$ and $U$ (high forces).

- Kinetically-stabilized non-native structures due to the simultaneous binding of two echinomycin ligands to dsDNA, misfolded: $N, M$.

2. Classification of trajectories.

Each trajectory can be classified as a function of the initial and final state. For instance, in EcoRI experiments we have four types of trajectories: $(i)$ start at $B$ and end at $U$; (ii) start at $B$ and end at $B$; (iii) start at $U$ and end at $B$; and (iv) start at $U$ and end at $U$.

3. Obtain partial work distributions.

In each cyclic trajectory, the work is calculated as the area enclosed in the trajectory. The partial work distribution is the histogram of work values restricted to each type of trajectory.

4. Obtain the grand-canonical partial partition function for the intial and the final state. The partial partition function for a state $S$ is computed in the restricted subset of configurations $\mathcal{C}_{j}$ that characterize state $S$ :

$$
Z_{S}^{G C}=\sum_{N_{i} \in S} \sum_{\mathcal{C}_{j}\left(N_{i}\right) \in S} z^{N_{i}} e^{-\beta E\left(\mathcal{C}_{j}\right)},
$$

where $N_{i}$ and $E\left(C_{i}\right)$ are the number of bound particles and the energy in configuration $C_{i}, z=e^{\beta \mu}$ is the fugacity, and $\mu$ is the chemical potential of the binding agent.

In the particular cases studied in this work these are:

- Specific binding of EcoRI and echinomycin to dsDNA.

$$
Z_{U}^{G C}=e^{-\beta G_{U}\left(\lambda_{0}\right)}, \quad Z_{B}^{G C}=z e^{-\beta\left(\varepsilon+G_{n}\left(\lambda_{0}\right)\right)}=e^{-\beta\left(\varepsilon-\mu+G_{n}\left(\lambda_{0}\right)\right)} .
$$

The term $G_{U}\left(\lambda_{0}\right)$ is the free energy of the hairpin in state $U$ at $\lambda_{0}$. The term $G_{n}\left(\lambda_{0}\right)$ is the free energy of the hairpin at $\lambda_{0}$ with $n$ unfolded basepairs in the 
hairpin stem prior to the binding site, $\varepsilon$ is the binding energy of the ligand and $\mu$ is its chemical potential. We refer to $\mu-\varepsilon$ as the binding free energy $\Delta G_{\text {bind }}$.

- Specific binding of a short oligo to its complementary ssDNA sequence.

$$
Z_{N}^{G C}=e^{-\beta G_{N}\left(\lambda_{0}\right)}, \quad Z_{B}^{G C}=z e^{-\beta\left(\varepsilon+G_{U}\left(\lambda_{0}\right)\right)}=e^{-\beta\left(\varepsilon-\mu+G_{U}\left(\lambda_{0}\right)\right)},
$$

where $G_{N}\left(\lambda_{0}\right)$ and $G_{U}\left(\lambda_{0}\right)$ are the free energies of the hairpin at $\lambda_{0}$ in the folded $(N)$ or unfolded $(U)$ state respectively, and $\Delta G_{\text {bind }}=\mu-\varepsilon$ is the binding free energy of the oligonucleotide.

- Non-specific binding of echinomycin to dsDNA.

$$
Z_{U}^{G C}=e^{-\beta G_{U}\left(\lambda_{0}\right)}, \quad Z_{B_{i}}^{G C}=z e^{-\beta\left(\varepsilon_{i}+G_{n_{i}}\left(\lambda_{0}\right)\right)}=e^{-\beta\left(\varepsilon_{i}-\mu+G_{n_{i}}\left(\lambda_{0}\right)\right)},
$$

where $B_{i}(i=1, \ldots, \mathcal{N})$ is a possible binding state (echinomycin binding to any site in dsDNA), $\varepsilon_{i}$ is the binding free energy at position $i$ and $G_{n_{i}}\left(\lambda_{0}\right)$ is the free energy of the hairpin at $\lambda_{0}$ where the $n_{i}$ basepairs prior to the position binding site of echinomycin are unfolded.

The FTLB applies to each pair os states $B_{i}$ and $U$, therefore:

$$
\sum_{i=1}^{\mathcal{N}} \frac{\phi^{B_{i} \rightarrow U}}{\phi^{U \rightarrow B_{i}}} \frac{P^{B_{i} \rightarrow U}(W)}{P^{U \rightarrow B_{i}}(-W)}=\sum_{i=1}^{\mathcal{N}} e^{\beta W} e^{-\beta\left(\mu-\varepsilon_{i}+\Delta G_{n_{i} U}\left(\lambda_{0}\right)\right)} .
$$

By assuming that $\phi^{B_{i} \rightarrow U}=\phi^{B \rightarrow U}$ and $\phi^{U \rightarrow B_{i}}=\frac{1}{\mathcal{N}} \phi^{U \rightarrow B}, \varepsilon_{i}=\varepsilon_{n}(i=1 \ldots \mathcal{N})$ and:

$$
\begin{gathered}
P^{B \rightarrow U}(W)=\frac{1}{\mathcal{N}} \sum_{i=1}^{\mathcal{N}} P^{B_{i} \rightarrow U}(W), \\
P^{U \rightarrow B}(-W) \simeq P^{U \rightarrow B_{i}}(-W), \quad \forall i,
\end{gathered}
$$

it can be shown that:

$$
\begin{aligned}
\frac{\phi^{B \rightarrow U}}{\phi^{U \rightarrow B}} \frac{P^{B \rightarrow U}(W)}{P^{U \rightarrow B}(-W)} & =e^{\beta W} \frac{e^{-\beta\left(\mu-\varepsilon_{n}\right)}}{\mathcal{N}^{2}} \sum_{i=1}^{\mathcal{N}} e^{-\beta \Delta G_{n_{i} U}\left(\lambda_{0}\right)} \\
& =e^{\beta\left(W-\Delta G_{B U}\right)}
\end{aligned}
$$

where $\Delta G_{B U}=\Delta G_{\text {bind }}-k_{\mathrm{B}} T \log \sum_{i=1}^{\mathcal{N}} e^{-\beta \Delta G_{n_{i} U}\left(\lambda_{0}\right)}+2 k_{\mathrm{B}} T \log \mathcal{N}$. 
- Simultaneous specific binding of two echinomycin molecules to sequential dsDNA sites (cooperativity).

$$
Z_{U}^{G C}=e^{-\beta G_{U}\left(\lambda_{0}\right)}, \quad Z_{B^{2}}^{G C}=z^{2} e^{-\beta\left(2 \varepsilon+G_{n}\left(\lambda_{0}\right)\right)},
$$

- Stabilization of misfolded states through simultaneous binding of two echinomycin molecules to sequential dsDNA sites.

$$
Z_{B}^{G C}=z^{2} e^{-\beta\left(2 \varepsilon+G_{N}\left(\lambda_{0}\right)\right)}, \quad \quad Z_{M}^{G C}=z^{\prime 2} e^{-\beta\left(2 \varepsilon^{\prime}+G_{M}\left(\lambda_{0}\right)\right)} .
$$

5. Plug everything into the FTLB.

According to Eq. (12), we write the FTLB for trajectories $A \rightarrow B(B \rightarrow A)$ as:

$$
\frac{\phi^{A \rightarrow B}}{\phi^{B \rightarrow A}} \frac{P^{A \rightarrow B}(W)}{P^{B \rightarrow A}(-W)}=e^{\beta W} \frac{Z_{B}^{G C}}{Z_{A}^{G C}}=e^{\beta\left(W-\Delta G_{A B}\right)},
$$

Use the Bennett acceptance ratio method for a better estimation of $\Delta G_{A B}$ (section 5 ).

6. Extract the elastic contributions to $\Delta G_{A B}$ in order to get the binding free energy $\Delta G_{\text {bind }}($ Section 5).

\section{Bennett acceptance ratio method}

The Bennett acceptance ratio method is used to estimate the free-energy difference $\Delta G_{A B}$ between two states that satisfies Eq. (12) from non-equilibrium work measurements. Given a set of $n_{F}\left(n_{R}\right)$ forward (reversed) work measurements $W_{i}$, it is shown in [40, 41] that the solution $u$ of the following transcendental equation:

$$
\frac{u}{k_{\mathrm{B}} T}=-\log \left(\frac{\phi^{A \rightarrow B}}{\phi^{B \rightarrow A}}\right)+z_{R}(u)-z_{F}(u),
$$

where:

$$
\begin{aligned}
z_{R}(u) & =\log \frac{1}{n_{R}} \sum_{i=1}^{n_{R}}\left(\frac{e^{-\beta W_{i}}}{1+\frac{n_{F}}{n_{R}} e^{-\beta\left(W_{i}+u\right)}}\right) \\
z_{F}(u) & =\log \frac{1}{n_{F}} \sum_{i=1}^{n_{F}}\left(\frac{1}{1+\frac{n_{F}}{n_{R}} e^{\beta\left(W_{i}-u\right)}}\right)
\end{aligned}
$$

minimizes the statistical variance of the free energy estimation for $u=\Delta G_{A B}$. 


\section{Energetic contributions to the binding free energy}

The free energy difference $\Delta G_{A B}$ obtained by using the FTLB contains the binding free energy of the ligand to the given substrate $\Delta G_{\text {bind }}$ plus elastic and thermodynamic energetic contributions of the experimental setup $\Delta G_{A B}\left(\lambda_{0}\right)$, which can be described as follows:

$$
\begin{aligned}
\Delta G_{A B}\left(\lambda_{0}\right) & =G_{B}\left(\lambda_{0}\right)-G_{A}\left(\lambda_{0}\right) \\
& =\Delta G_{A B}^{0}+\Delta W_{A B}^{\text {handles }}+\Delta W_{A B}^{\text {bead }}+\Delta W_{A B}^{\text {ssDNA }}+\Delta W_{A B}^{\mathrm{d}} .
\end{aligned}
$$

Here, $A(B)$ stands for the configuration of the hairpin at the beginning (ending) of the cyclic protocol at $\lambda_{0}$. In what follows, $f_{A}\left(f_{B}\right)$ is the force acting on the molecular setup when the hairpin is in state $A(B)$ at $\lambda_{0}$.

The term $\Delta G_{A B}^{0}=G_{B}^{0}-G_{A}^{0}$ is the difference between the free energy of formation of the conformations of the DNA hairpin in states $A$ and $B$. This term depends on the sequence of the hairpin and is usually calculated using the nearest-neighbor model and the unified oligonucleotide set of basepair free energies [29, 39] or can be recovered from pulling experiments performed in the absence of binding agents using fluctuation relations [15].

The two terms $\Delta W_{A B}^{\text {handles }}$ and $\Delta W_{A B}^{\text {bead }}$ correspond to the reversible work needed to stretch the handles and move the bead captured in the optical trap from state $A$ to state $B$. For short handles:

$$
\Delta W_{A B}^{\text {handles }}+\Delta W_{A B}^{\text {bead }}=\frac{f_{B}^{2}-f_{A}^{2}}{2 k_{\mathrm{eff}}},
$$

where $k_{\text {eff }}$ is the effective stiffness of the experimental setup, equal to the slope of the forcedistance curve measured in the force-branch corresponding to the native state of the hairpin. For long handles:

$$
\Delta W_{A B}^{\text {handles }}+\Delta W_{A B}^{\text {bead }}=\int_{x_{\mathrm{h}}\left(f_{A}\right)}^{x_{\mathrm{h}}\left(f_{B}\right)} f\left(x^{\prime}\right) d x^{\prime}+\frac{f_{B}^{2}-f_{A}^{2}}{2 k_{b}}
$$

where $x_{\mathrm{h}}\left(f_{A}\right)\left(x_{\mathrm{h}}\left(f_{B}\right)\right)$ is the equilibrium end-to-end distance of the handles at force $f_{A}\left(f_{B}\right)$, which is calculated according to the worm-like chain model using a persistence length equal to $43.7 \mathrm{~nm}$, a contour length equal to $446.08 \mathrm{~nm}$ and a Young modulus of $1280 \mathrm{pN}$ [43]; and $k_{b}=0.068 \mathrm{pN} / \mathrm{nm}$ is the stiffness of the optical trap in our setup [36].

The term $\Delta W_{A B}^{\text {ssDNA }}=W_{B}^{\text {ssDNA }}-W_{A}^{\text {ssDNA }}$ corresponds to the difference between the reversible work needed to stretch the released single stranded DNA in configurations $B$ and 
$A$ from zero force to $f_{A}$ and $f_{B}$, respectively. This is calculated according to:

$$
\Delta W_{A B}^{\mathrm{ssDNA}}=\int_{0}^{x_{\mathrm{ssDNA}}\left(f_{B}\right)} f\left(x^{\prime}\right) d x^{\prime}-\int_{0}^{x_{\mathrm{sSNA}}\left(f_{A}\right)} f\left(x^{\prime}\right) d x^{\prime},
$$

where equilibrium relation between the force and the end-to-end distance $f(x)$ and its inverse $x_{\mathrm{ssDNA}}(f)$ are modeled according to the worm-like chain ideal elastic model with a persistence length equal to $1.35 \mathrm{~nm}$ and an inter-phosphate distance of $0.59 \mathrm{~nm} / \mathrm{base}$, and the number of bases released as single-stranded DNA depends on state $B$ or $A$ [44].

The term $\Delta W_{A B}^{\mathrm{d}}$ is the difference between reversible work needed to orient the doublehelix diameter between states $A$ and $B$ :

$$
\Delta W_{A B}^{\mathrm{d}}=\int_{0}^{x_{\mathrm{d}}\left(f_{B}\right)} f\left(x^{\prime}\right) d x^{\prime}-\int_{0}^{x_{\mathrm{d}}\left(f_{A}\right)} f\left(x^{\prime}\right) d x^{\prime} .
$$

The helix diameter is modeled as a single bond of length $d=2 \mathrm{~nm}$ that is oriented due to the action of an external force $f$ [36, 44].

\section{Equilibrium experiments for the hairpin-oligonucleotide system}

In equilibrium experiments in passive-mode the position of the optical trap is held constant. Hairpin "Oligo" (section 5) hops rapidly between the unfolded (low forces) and the folded (high forces) states (Fig. 4a) [36]. The binding and unbinding of the 10-bp oligonucleotide to its complementary sequence in the hairpin occur at a slower timescale (several seconds), and consequently binding/unbinding events can be readily identified in experiments in which a concentration of binding oligonucleotide is present (Fig. 4 p).

To extract the binding free energy of the oligo to the unfolded DNA hairpin we consider the reaction pathway $N \leftrightarrows U \leftrightarrows B$, where $N$ corresponds to the state where the hairpin is in its native state, $U$ corresponds to the state where the hairpin is unfolded (and the oligo is not bound), and $B$ corresponds to the state where the hairpin is unfolded and an oligo bound. Since in equilibrium experiments we cannot distinguish between states $U$ and $B$ due to the very similar extension of dsDNA and ssDNA at the relevant range of forces for these experiments (Fig. 4b) [42], we define the joint probability $\rho_{U B}=\rho_{U}+\rho_{B}$. By considering that detailed balance is verified, it can be shown that:

$$
\beta \Delta G_{\mathrm{bind}}=\log \left(\frac{\rho_{U B}}{\rho_{N}} e^{\beta \Delta G_{N U}}-1\right)+\beta \Delta G_{U B} .
$$


In Fig. 5 we show force-time trajectories with the corresponding experimental probability density functions obtained at different values of $\lambda$ at $400 \mathrm{nM}$ oligo. From the fit to a double Gaussian (blue dashed line) we can extract the weights $\rho_{N}$ and $\rho_{U B}$, and determine the two forces levels $\left(f_{N}\right.$ and $\left.f_{U}=f_{B}\right)$ as the average force of each Gaussian peak. In Table VII we summarize the different contributions to extract $\Delta G_{\text {bind }}$ for three different experimental traces. Since $f_{U}=f_{B}$, the terms $\Delta W_{U B}^{\text {handles }}, \Delta W_{U B}^{\text {bead }}$ and $\Delta W_{U B}^{\text {d }}$ equal zero.

In average, we find that at a concentration of $400 \mathrm{nM}$ oligo $\langle\beta(\mu-\varepsilon)\rangle_{400 \mathrm{nM}[\text { oligo }]}=7 \pm 1$, in good agreement with the theoretical predictions and with the results obtained by applying the FTLB in non-equilibrium pulling experiments. 


\section{SUPPLEMENTARY FIGURES}

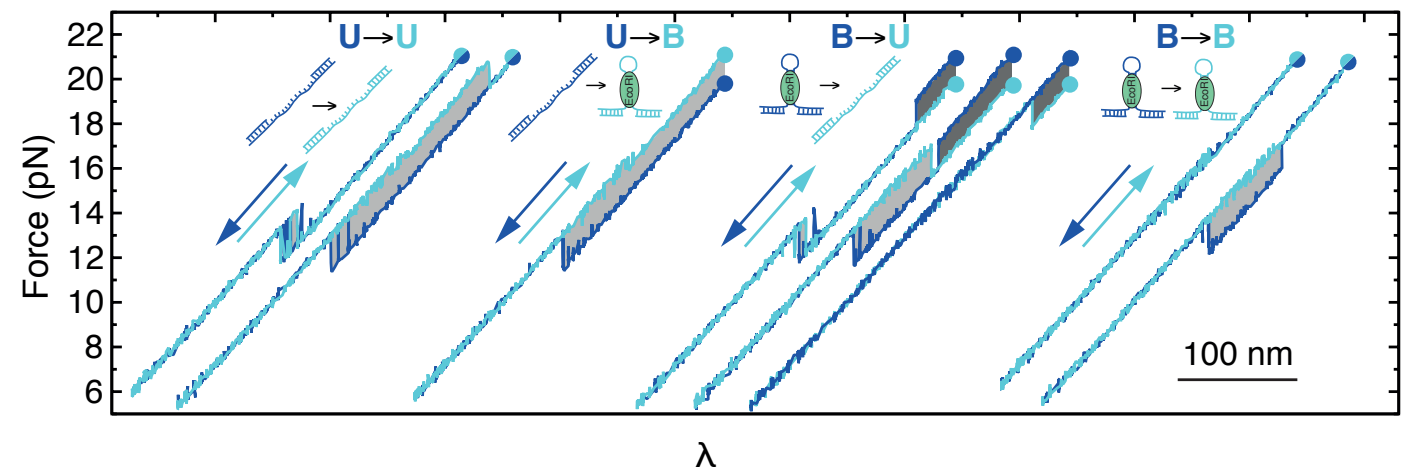

Figure 1. Force-distance curves of EcoRI binding to DNA. Example of cyclic pulling curves classified according to their initial (blue dot) and final state (cyan dot) that start and end at a high force $(\sim 21 \mathrm{pN})$. Work is calculated by integrating the area between the two curves and is shown in dark/light gray for positive/negative work values. 

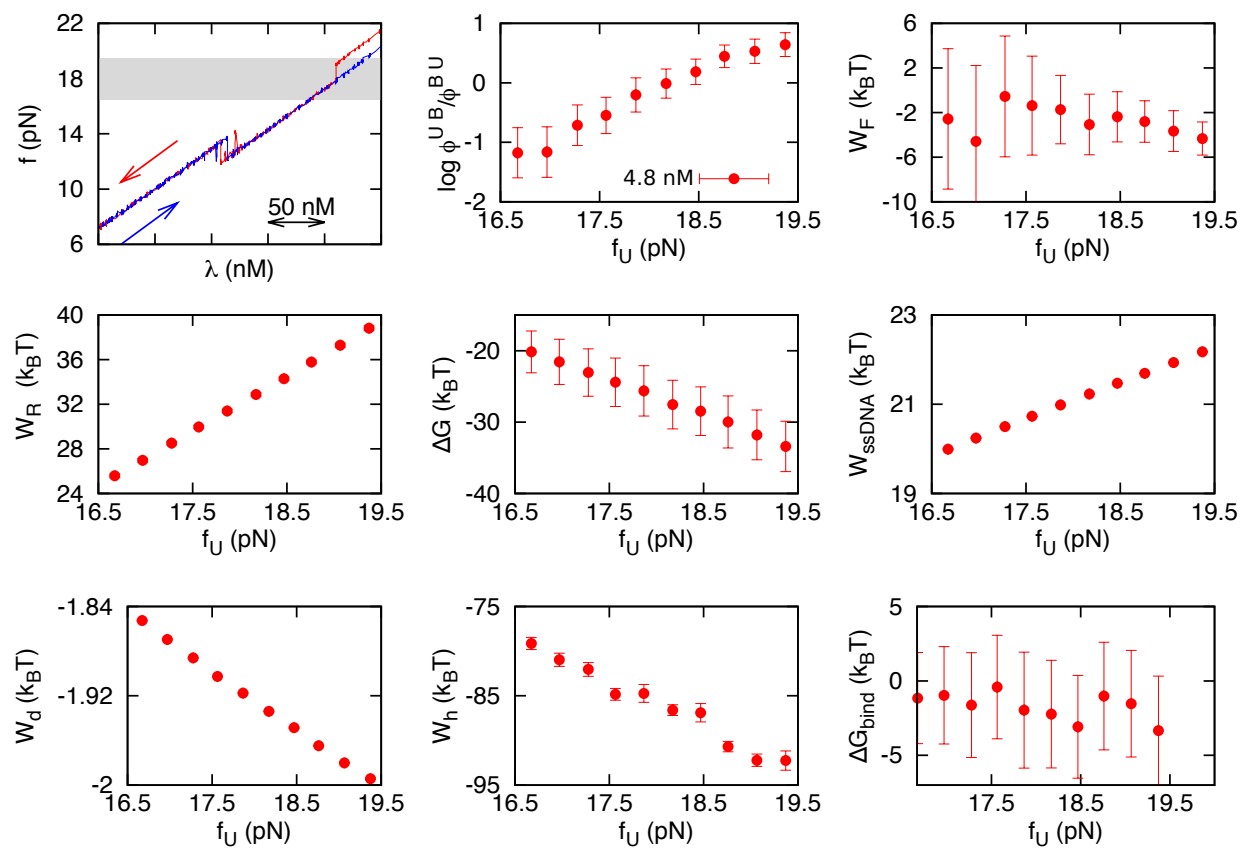

Figure 2. Effect of the upper unfolding force on the derivation of the binding free energy.

The intitial/final value of $\lambda$ can be set to any position where we can unambiguously distinguish states $B$ and $U$. For each value of $\lambda$, a corresponding force $f_{U}$ is observed in the unfolded branch (gray area in top-left panel). We then apply the FTLB to extract the binding energy of EcoRI for different positions of $\lambda$ at high forces. The rest of the panels show the dependence on force $f_{U}$ of the prefactor $\log \left(\phi^{U \rightarrow B} / \phi^{B \rightarrow U}\right)$; the forward and reversed mean work values $\left\langle W_{F}\right\rangle$ and $\left\langle W_{R}\right\rangle$; the free energy difference recovered with the direct application of the FTLB, $\Delta G$; the contribution to $\Delta G$ due to the released ssDNA, the hairpin diameter and the handles of the system $W_{\mathrm{ssDNA}}$, $W_{\mathrm{d}}$ and $W_{\mathrm{h}}$ respectively; and finally, the free energy of binding $\Delta G_{\text {bind }}$. It can be seen that the resulting value of $\Delta G_{\text {bind }}$ does not depend on $f_{U}$, therefore does not depend on the initial/final position of $\lambda$ in the cyclic pulling protocol. Data shown corresponds to experiments performed at $4.8 \mathrm{nM}$ for one molecule where a total of 413 cycles where recorded. 


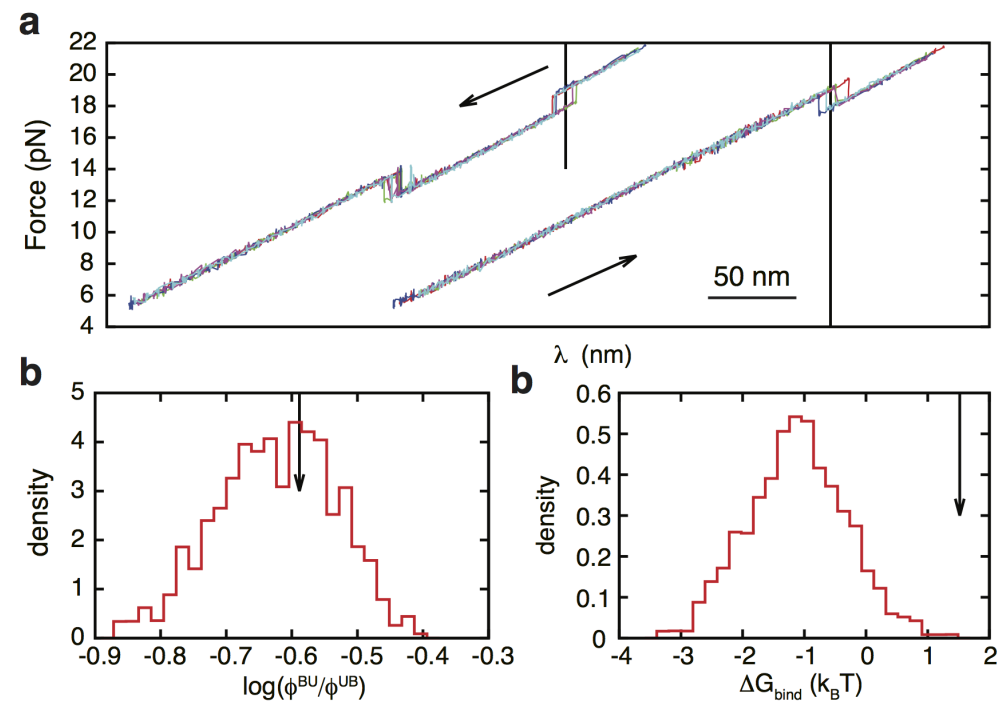

Figure 3. Effect of random classification of $B, U$ states in transitions close to the initial/final $\lambda$. (a) Example of folding (left) and unfolding (right) force-distance curves where a transition $B \rightarrow U$ occurs close to the initial/final value of $\lambda$ (indicated with a vertical black line respectively). Probabily density function of values for $k_{\mathrm{B}} T \log \left(\phi^{U \rightarrow B} / \phi^{B \rightarrow U}\right)$ (b) and $\Delta G_{\text {bind }}$ (c) obtained by of randomly assigning 500 independent times states $\mathrm{B}$ and $\mathrm{U}$ to trajectories where an unbinding transition is observed at $\lambda \pm 10 \mathrm{~nm}$ (such as the ones depicted in panel a). Vertical arrows indicate the value recovered with the correct classification of the initial/final states along the cyclic pulling protocol. We observed that a random classification of states in trajectories were transitions are observed close the the inifial/final value of $\lambda$ persistently leads to lower values for the binding free energy. 


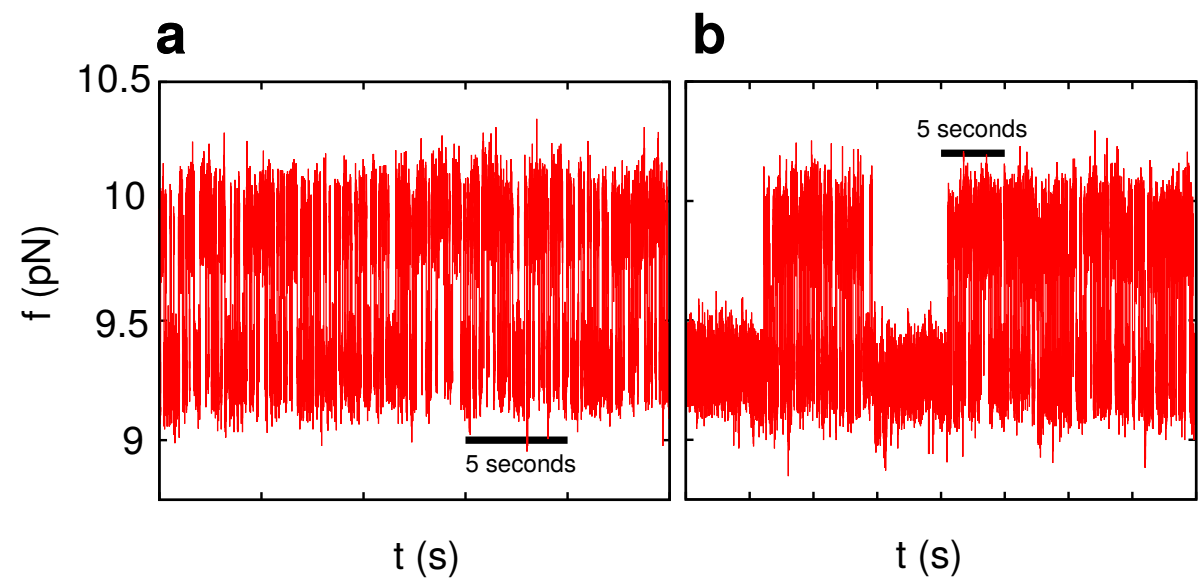

Figure 4. Experimental traces of equilibrium experiments of oligonucleotide binding. (a) Equilibrium experiments performed without oligo with hairpin "Oligo". (b) Equilibrium experiments performed at $400 \mathrm{nM}$ [oligo]. Two time-scales are revealed when the hairpin is in the unfolded state (low forces). 

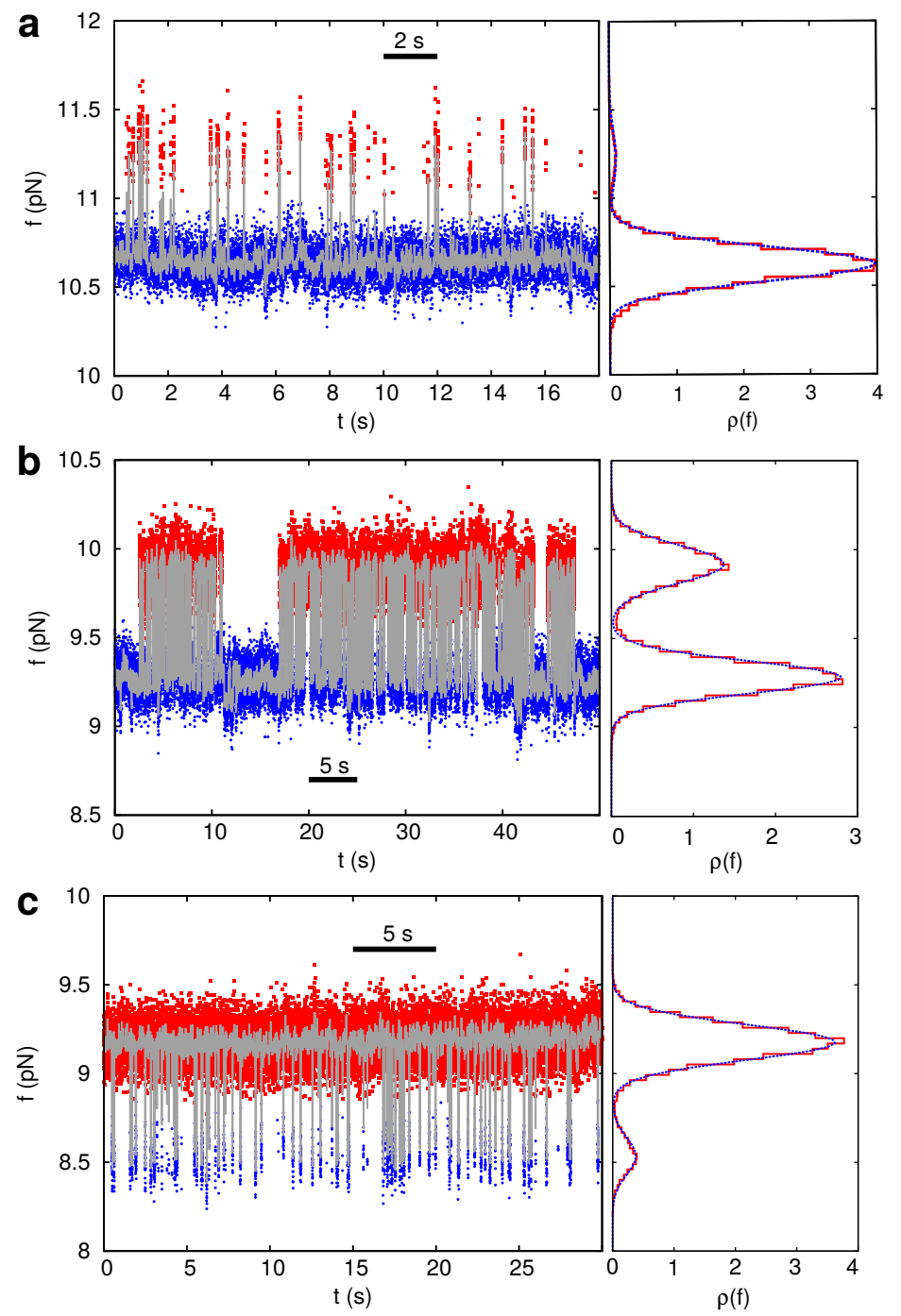

Figure 5. Equilibrium experiments at different forces. In gray we show an averaged experimental trace; in red we highlight experimental data points (acquisition rate: $1 \mathrm{kHz}$ ) where the hairpin is in the folded state (and therefore no oligo is bound), whereas in blue we highlight data points where the hairpin is in the unfolded state (either with the oligo bound or not bound). Two time-scales are observed in the experiments showing that the oligo binds and unbinds from the hairpin in an stochastic manner and with a timescale much longer than the folding/unfolding rate of the hairpin. The panels on the right show an histogram of the probability density (red) and a double gaussian fit to the data (blue). 


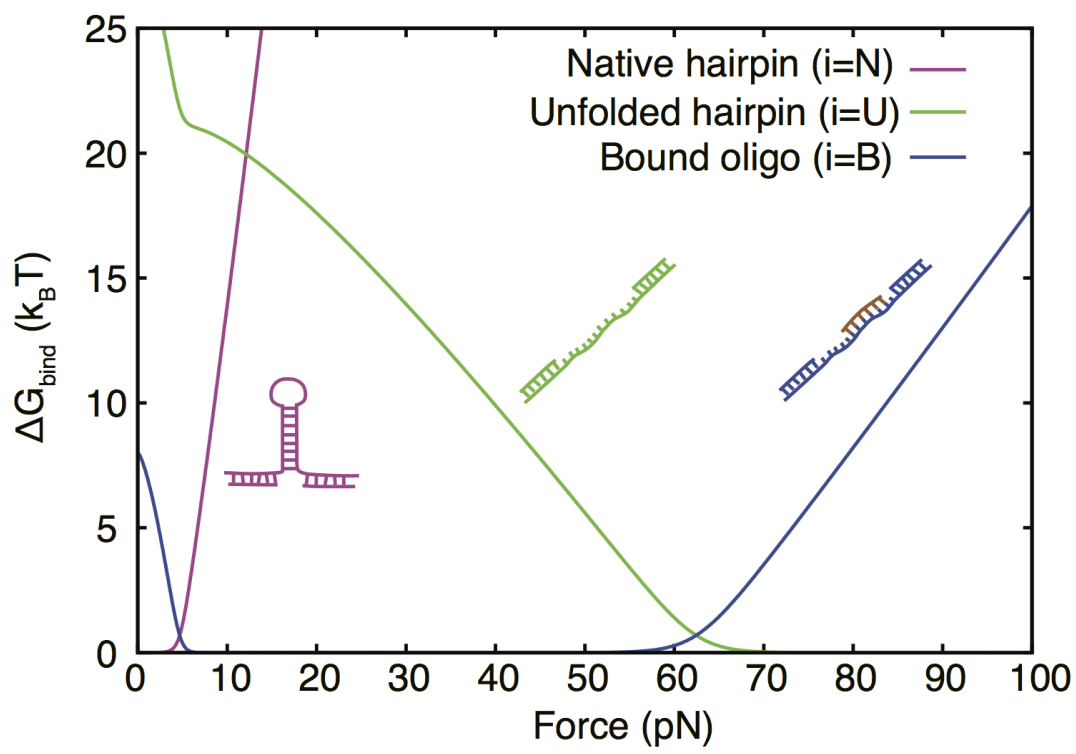

Figure 6. Free energy of branches $B, N$ and $U$. Free energy branches are shown as a function of force and computed relative to the total free energy of the system (also called potential of mean force, equal to $\left.-\log \left[\exp \left(-\beta \Delta G_{B}\right)+\exp \left(-\beta \Delta G_{N}\right)+\exp \left(-\beta \Delta G_{U}\right)\right]\right)$. The free energy of state $B$ is computed by taking into account the free energy of binding (equal to $20 k_{\mathrm{B}} T$ ) plus the elastic response of a DNA made of two ligated ssDNA and dsDNA segments. The first segment is a 24 bases-long ssDNA chain (modeled with the worm-like chain (WLC) model with persistence length equal to $1.5 \mathrm{~nm}$ and inter-phosphate distance equal to $0.59 \mathrm{~nm} /$ base). The second segment is the elastic response of a 10 basepair-long dsDNA chain (modeled with the WLC model with persistence length equal to $50 \mathrm{~nm}$ and inter-phosphate distance equal to $0.34 \mathrm{~nm} / \mathrm{base})$. The free energy of state $U$ is computed by taking only into account the elastic response of a 34 bases-long ssDNA chain. Finally, the free energy of state N contains the folding free energy of the hairpin $\left(28 k_{\mathrm{B}} T\right)$ plus the elastic response of the hairpin diameter, modeled as a bond of length $2.0 \mathrm{~nm}$ (equal to the hairpin diameter) that is oriented in the presence of a force. It can be seen that at low forces state $N$ is the most stable. However, at $\sim 5 \mathrm{pN}$ state $B$ becomes the most stable until $\sim 62 \mathrm{pN}$, where state $U$ becomes more stable. Therefore, with this simple model we predict that the threshold force above which the oligo will not bind is $\sim 62 \mathrm{pN}$. However, at those high forces potential perturbations of the force into the ssDNA structure neglected in the model might change this value. 

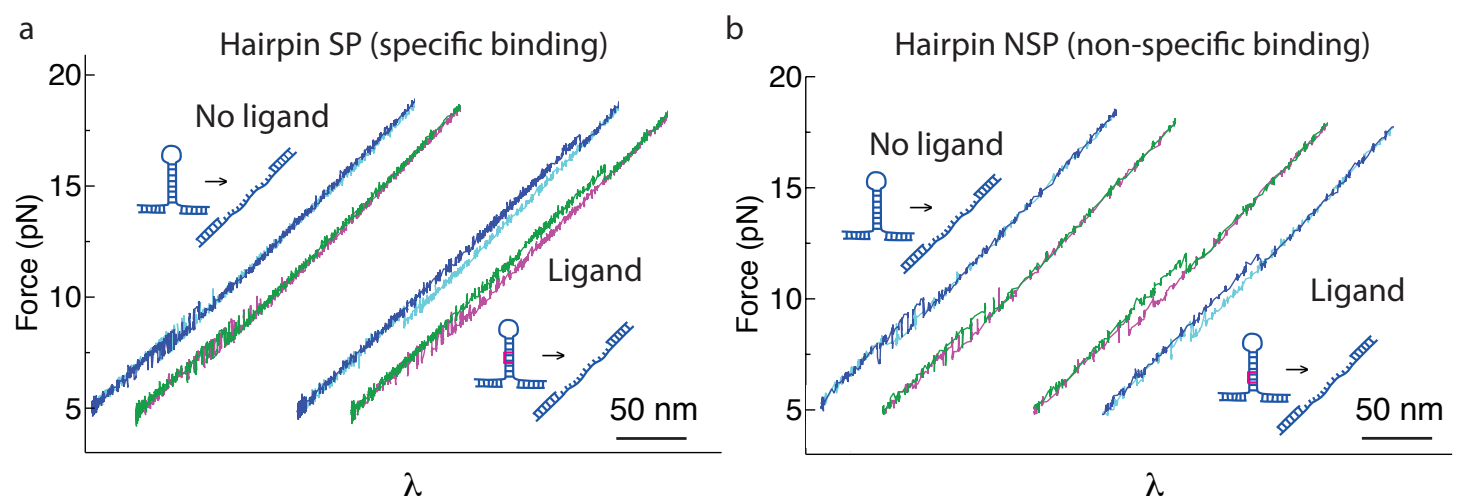

Figure 7. Force-distance curves of hairpins SP and NSP. (a) FDCs of hairpin SP in the absence (left) and presence of ligand (right). (b) FDCs of hairpin NSP in the absence (left) and presence of ligand (right). In each FDC blue/green is unfolding and cyan/magenta refolding. Pulling speed is $70 \mathrm{~nm} / \mathrm{s}$ in (a) and $250 \mathrm{~nm} / \mathrm{s}$ in (b). 

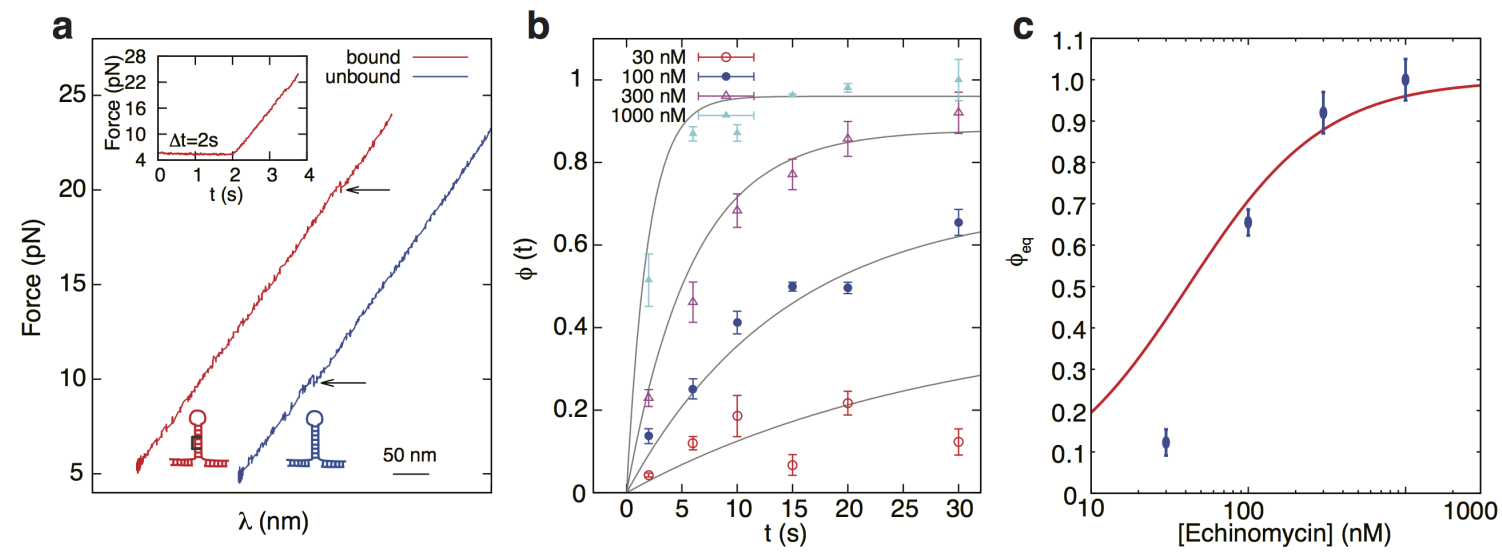

Figure 8. Specific ligand binding of echinomycin to DNA. (a) Fast mechanical unfolding is performed after incubating the hairpin at $\sim 5 \mathrm{pN}$ during a fixed time interval $\Delta t$. The unfolding force of the hairpin, indicated with an arrow, relates to the bound state of echinomycin: unfolding forces above (below) $15 \mathrm{pN}$ (red(blue) curve) indicate that an echinomycin molecules has (has not) bound to the hairpin (Fig. 3a, main document). (b) Fraction of bound states as a function of the time $\Delta t$ and concentration of echinomycin and fit to to a first order reaction kinetics model $(\mathrm{DNA}+\mathrm{I} \rightleftarrows \mathrm{DNA} \cdot \mathrm{I})$ where $\phi(t)=\frac{k_{\rightarrow}[I]}{k_{\leftarrow}[I]+k_{\leftarrow}}\left(1-\exp \left[\left(k_{\rightarrow}[I]+k_{\leftarrow}\right) t\right]\right)$. From the fit, we obtain $k_{\rightarrow}=$ $(4.9 \pm 0.4) \times 10^{-4} \mathrm{nM}^{-1} \mathrm{~s}^{-1}$ and $k_{\leftarrow}=(2.0 \pm 0.5) \times 10^{-2} \mathrm{~s}^{-1}$, which implies $K_{d}=k_{\leftarrow} / k_{\rightarrow}=41 \pm 10$ $\mathrm{nM}$ and $\Delta G_{\text {bind }}=17 \pm 1 k_{\mathrm{B}} T$. This result is in good agreement with the value obtained using the FTLB $\left(\Delta G_{\text {bind }}=20 \pm 1 k_{\mathrm{B}} T\right)$. (c) Binding isotherm of echinomycin determined from optical trapping. The red curve has been obtained from the fit in panel b. Blue points are the fraction of bound population measured as described in panel a at the largest measured time $\Delta t=30 \mathrm{~s}$. The disagreement between theory and experiments observed at low concentrations shows that binding kinetics is still out of equilibrium at the largest measured time of 30s. Error bars are standard errors computed by averaging over different molecules. 

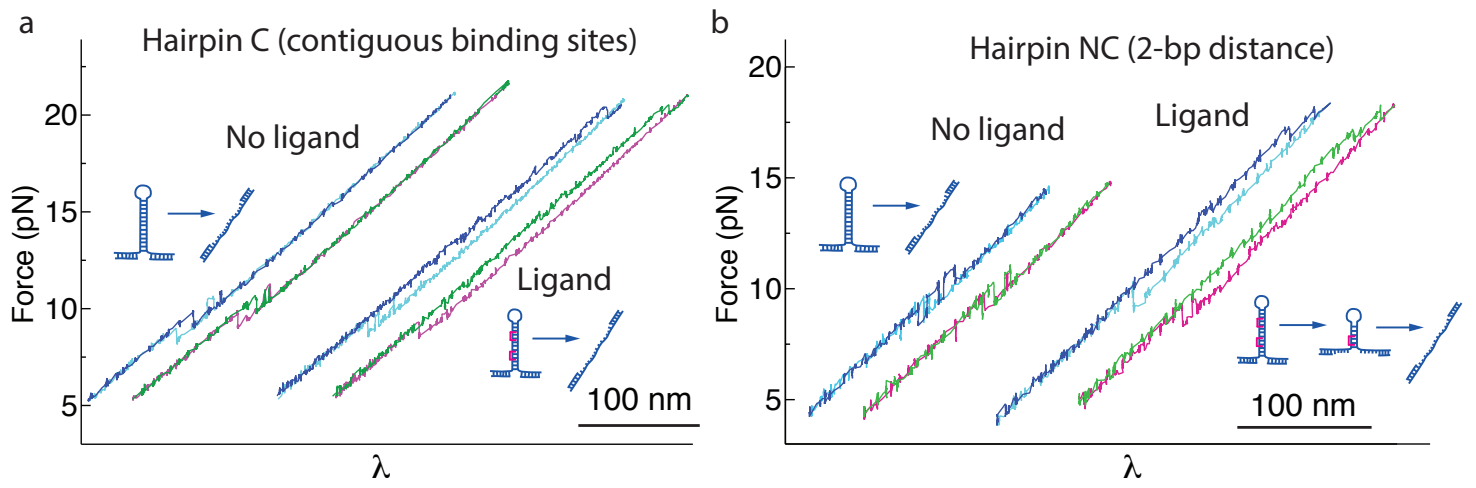

Figure 9. Force-distance curves of hairpins $\mathbf{C}$ and NC. (a) FDCs of hairpin $\mathrm{C}$ in the absence (left) and presence of ligand (right) (b) FDCs of hairpin NC in the absence (left) and presence of ligand. In the presence of ligand, FDCs of hairpin $\mathrm{C}$ show higher unfolding forces than those of hairpin $\mathrm{NC}$, in agreement with the proposed cooperative effect between ligand pairs. Similarly a partially unfolded intermediate with just one ligand bound is observed in hairpin NC, whereas hairpin $\mathrm{C}$ cooperatively unfolds in a single step. In each FDC blue/green is unfolding and cyan/magenta refolding. Pulling speed is $70 \mathrm{~nm} / \mathrm{s}$ in (a) and $250 \mathrm{~nm} / \mathrm{s}$ in (b). 


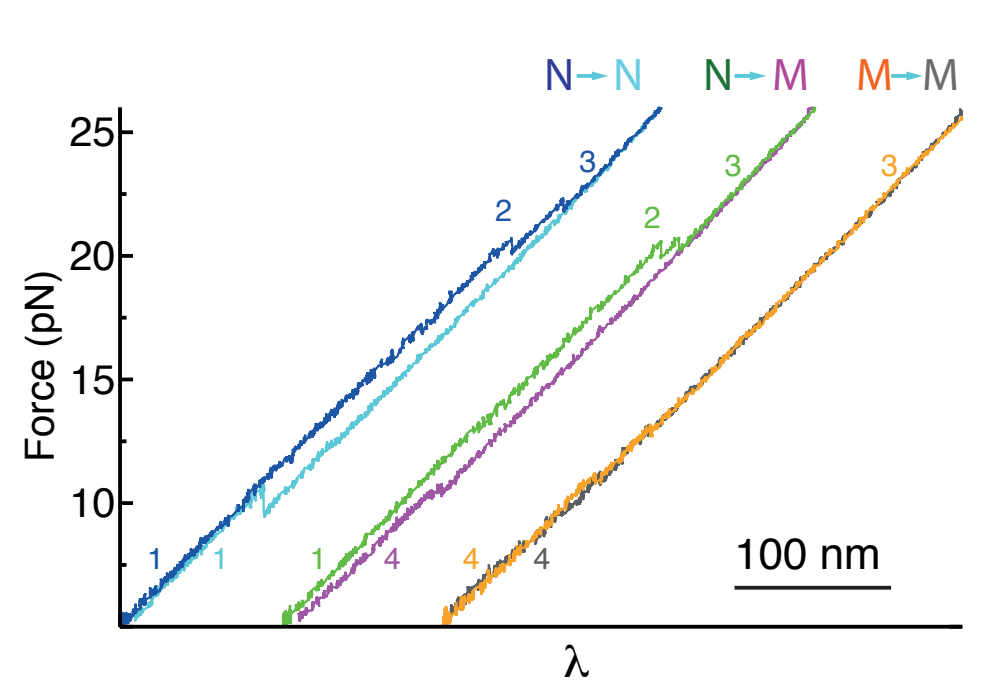

1. Native

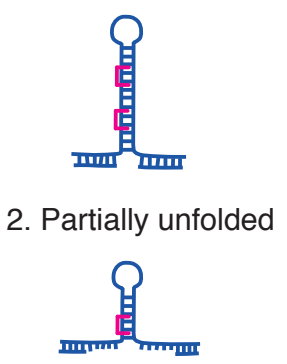

3. Unfolded

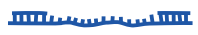

4. Misfolded

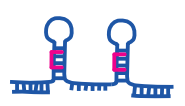

Figure 10. Force-distance curves of hairpin $\mathbf{M}$ at $[$ Echinomycin] $=10 \mu \mathbf{M}$. The ligand can kinetically trap a misfolded state consisting of two 4-bp DNA hairpins serially connected. Characteristic pulling curves connecting the native $(\mathrm{N})$ and misfolded $(\mathrm{M})$ state are shown. The different molecular configurations observed during the pulling curve are indicated in the scheme. Pulling speed is $70 \mathrm{~nm} / \mathrm{s}$. 


\section{SUPPLEMENTARY TABLES}

\begin{tabular}{lll}
\hline \hline [EcoRI] $(\mathrm{nM})$ & pulled molecules (number of cycles per molecule) & total cycles $N$ \\
\hline 0.25 & $5(141,87,95,360,101)$ & 784 \\
0.50 & $8(297,93,43,101,40,18,137,27)$ & 756 \\
1.00 & $8(852,555,486,48,153,76,159,404)$ & 2733 \\
2.40 & $8(170,356,350,433,212,93,245,126)$ & 1985 \\
4.80 & $3(413,339,349)$ & 1101 \\
10.0 & $10(470,317,290,242,87,200,248,401,102,396)$ & 2753 \\
20.0 & $3(257,242,668)$ & 1167 \\
\hline
\end{tabular}

Table I. Number of experiments performed at $130 \mathrm{mM} \mathrm{NaCl}$ for different concentrations

of EcoRI. Number of molecules measured at each concentration of EcoRI, corresponding cycles per molecule shown in parenthesis, and total number of cycles used for computing the binding energy. 


\begin{tabular}{|c|c|c|c|c|c|c|}
\hline$f_{B}(\mathrm{pN}) \quad f_{U}(\mathrm{pN})$ & $\Delta G_{n}^{0}$ & $\Delta W_{n U}^{\text {hand }}$ & lles $+\Delta W_{n U}^{\text {bead }}$ & $\Delta W_{i}$ & sDNA & $\Delta W_{n U}^{d}$ \\
\hline $19.77 \pm 0.01$ & $18.54 \pm 0.01 \quad 42 \pm 2$ & \multicolumn{2}{|r|}{$-92 \pm 1$} & \multicolumn{3}{|c|}{$21.51 \pm 0.01-1.955 \pm 0.001$} \\
\hline$[$ EcoRI $](n M)$ & $\phi^{B \rightarrow U}$ & $\phi^{U \rightarrow B}$ & $\log \left(\phi^{B \rightarrow U} / \phi\right.$ & $(U \rightarrow B)$ & $\Delta G_{B U}$ & $\Delta G_{\text {bind }}$ \\
\hline 0.25 & $0.4 \pm 0.1$ & $0.12 \pm 0.03$ & $1.2 \pm 0.8$ & & $-29 \pm 2$ & $2 \pm 4$ \\
\hline 0.50 & $0.24 \pm 0.05$ & $0.22 \pm 0.02$ & $0.1 \pm 0.2$ & & $-25 \pm 2$ & $5 \pm 4$ \\
\hline 1.00 & $0.15 \pm 0.03$ & $0.31 \pm 0.02$ & $-0.8 \pm 0$ & & $-23 \pm 1$ & $5 \pm 3$ \\
\hline 2.40 & $0.24 \pm 0.04$ & $0.26 \pm 0.03$ & $-0.2 \pm 0$ & & $-24 \pm 2$ & $8 \pm 2$ \\
\hline 4.80 & $0.24 \pm 0.04$ & $0.27 \pm 0.03$ & $-0.1 \pm 0$ & & $-27 \pm 2$ & $5 \pm 3$ \\
\hline 10.0 & $0.17 \pm 0.02$ & $0.71 \pm 0.05$ & $-1.5 \pm 0$. & & $-22 \pm 1$ & $8 \pm 1$ \\
\hline 20.0 & $0.13 \pm 0.02$ & $0.83 \pm 0.03$ & $-1.9 \pm 0$. & & $-23 \pm 1$ & $8 \pm 1$ \\
\hline
\end{tabular}

Table II. Contributions to the binding free energy of EcoRI to dsDNA as a function of [EcoRI] at $130 \mathrm{mM} \mathrm{NaCl}$. For all the pulling experiments performed for different molecules at different concentrations of EcoRI, the initial/final value of the control parameter $\lambda$ was chosen so that forces $f_{B}$ and $f_{U}$ are on average the same. Hence, numerical values for $\Delta G_{n U}^{0}$, $\Delta W_{n U}^{\text {handles }}+\Delta W_{n U}^{\text {bead }}\left(\right.$ Eq. 27), $\Delta W_{n U}^{\text {ssDNA }}$ (Eq. 28), and $\Delta W_{n U}^{\text {d }}$ (Eq. 29) are also on average the same for different molecules pulled at different concentrations of EcoRI. In contrast, $\phi^{B \rightarrow U}, \phi^{U \rightarrow B}$, $\log \left(\phi^{B \rightarrow U} / \phi^{U \rightarrow B}\right), \Delta G_{B U}$ and $\Delta G_{\text {bind }}$ depend on the concentration of ligand. Here $n=7$. 


\begin{tabular}{llc}
\hline \hline$[\mathrm{NaCl}](\mathrm{mM})$ & pulled molecules (number of cycles per molecule) & total cycles $N$ \\
\hline 60 & $15(156,69,525,60,61,35,21,43,713,258,433,58,78,162,83)$ & 2755 \\
75 & $6(263,312,519,506,156,302)$ & 2058 \\
100 & $7(276,273,516,577,128,494,235)$ & 2499 \\
130 & $8(852,555,486,48,153,76,159,404)$ & 2733 \\
180 & $8(654,323,88,298,61,423,211,80)$ & 2138 \\
\hline
\end{tabular}

Table III. Number of experiments performed at $1 \mathrm{nM}$ EcoRI for different concentrations of $\mathrm{NaCl}$. 


\begin{tabular}{ccccc}
\hline \hline$f_{B}(\mathrm{pN})$ & $f_{U}(\mathrm{pN})$ & $\Delta W_{n U}^{\text {handles }}+\Delta W_{n U}^{\text {bead }}$ & $\Delta W_{n U}^{\text {ssDNA }}$ & $\Delta W_{n U}^{d}$ \\
\hline $19.76 \pm 0.01$ & $18.56 \pm 0.01$ & $-91 \pm 1$ & $21.54 \pm 0.01$ & $-1.955 \pm 0.001$
\end{tabular}

\begin{tabular}{ccccccc}
\hline \hline EcoRI $](\mathrm{nM})$ & $\Delta G_{n U}^{0}$ & $\phi^{B \rightarrow U}$ & $\phi^{U \rightarrow B}$ & $\log \left(\phi^{B \rightarrow U} / \phi^{U \rightarrow B}\right)$ & $\Delta G_{B U} \Delta G_{\text {bind }}$ \\
\hline 60 & $39 \pm 2$ & $0.056 \pm 0.008$ & $0.8 \pm 0.1$ & $-2.5 \pm 0.4$ & $-19 \pm 3$ & $13 \pm 3$ \\
75 & $40 \pm 2$ & $0.06 \pm 0.01$ & $0.7 \pm 0.1$ & $-2.5 \pm 0.4$ & $-20 \pm 1$ & $11 \pm 2$ \\
100 & $41 \pm 2$ & $0.14 \pm 0.02$ & $0.29 \pm 0.05$ & $-0.9 \pm 0.2$ & $-25 \pm 2$ & $5 \pm 2$ \\
130 & $42 \pm 2$ & $0.15 \pm 0.03$ & $0.31 \pm 0.02$ & $-0.8 \pm 0.1$ & $-23 \pm 1$ & $6 \pm 1$ \\
180 & $44 \pm 2$ & $0.44 \pm 0.07$ & $0.06 \pm 0.02$ & $1.6 \pm 0.4$ & $-27 \pm 2$ & $0 \pm 2$ \\
\hline
\end{tabular}

Table IV. Contributions to the binding free energy of EcoRI to dsDNA as a function of $[\mathrm{NaCl}]$ at $1 \mathbf{n M}$ EcoRI. Caption as in Table II 


\begin{tabular}{lll}
\hline \hline [oligo] $(\mathrm{nM})$ & pulled molecules (number of cycles per molecule) & total cycles $N$ \\
\hline 25 & $2(25,244)$ & 269 \\
50 & $7(57,131,72,242,311,9846)$ & 957 \\
100 & $6(182,198,22,435,368,386)$ & 1591 \\
200 & $5(28,17,59,67,87)$ & 258 \\
400 & $4(264,123,213,109)$ & 709 \\
1000 & $10(59,56,288,293,325,224,83,432,317)$ & 2077 \\
2000 & $5(185,603,74,273,147)$ & 1282 \\
\hline
\end{tabular}

Table V. Number of experiments performed at different concentrations of oligo. 


\begin{tabular}{ccccccc}
\hline \hline$f_{B}(\mathrm{pN})$ & $f_{N}(\mathrm{pN})$ & $\Delta G_{U N}^{0}$ & $\Delta W_{U N}^{\text {handles }}+\Delta W_{U N}^{\text {bead }}$ & $\Delta W_{U N}^{\text {ssDNA }}$ & $\Delta W_{U N}^{d}$ \\
\hline $6.20 \pm 0.06$ & $6.80 \pm 0.06$ & $-27.5 \pm 0.8$ & $16.5 \pm 0.5$ & $-4.81 \pm 0.05$ & $0.896 \pm 0.008$ \\
\hline \hline [oligo $(\mathrm{nM})$ & $\phi^{B \rightarrow N}$ & $\phi^{N \rightarrow B}$ & $\log \left(\phi^{B \rightarrow N} / \phi^{N \rightarrow B}\right)$ & $\Delta G_{B N}$ & $\Delta G_{\text {bind }}$ \\
\hline 25 & $1.0^{*}$ & $0.030 \pm 0.004$ & $3.6 \pm 0.1$ & $-11.3 \pm 0.7$ & $4 \pm 1$ \\
50 & $0.96 \pm 0.04$ & $0.037 \pm 0.006$ & $3.4 \pm 0.2$ & $-11.8 \pm 0.6$ & $3 \pm 1$ \\
100 & $0.94 \pm 0.03$ & $0.062 \pm 0.006$ & $2.79 \pm 0.08$ & $-11.0 \pm 0.6$ & $4 \pm 1$ \\
200 & $0.87 \pm 0.08$ & $0.09 \pm 0.03$ & $2.3 \pm 0.3$ & $-9.2 \pm 0.4$ & $6 \pm 1$ \\
400 & $0.86 \pm 0.06$ & $0.18 \pm 0.03$ & $1.6 \pm 0.3$ & $-8.4 \pm 0.3$ & $7 \pm 1$ \\
1000 & $0.49 \pm 0.05$ & $0.45 \pm 0.04$ & $0.37 \pm 0.07$ & $-7.7 \pm 0.5$ & $7 \pm 1$ \\
2000 & $0.37 \pm 0.02$ & $0.60 \pm 0.04$ & $-0.5 \pm 0.1$ & $-6.5 \pm 0.8$ & $8 \pm 1$ \\
\hline
\end{tabular}

Table VI. Contributions to the binding free energy of oligo to complementary ssDNA as a function of the concentration of the oligo. For all the pulling experiments performed for different molecules at different concentrations of oligo, the initial/final value of the control parameter $\lambda$ was chosen so that forces $f_{B}$ and $f_{N}$ are on average the same same. Numerical values for $\Delta G_{U N}^{0}, \Delta W_{U N}^{\text {handles }}+\Delta W_{U N}^{\text {bead }}$ (Eq. 27), $\Delta W_{U N}^{\text {ssDNA }}$ (Eq. 28), and $\Delta W_{U N}^{\mathrm{d}}$ (Eq. 29) are also on average the same for different molecules pulled at different concentrations of oligo. In contrast, $\phi^{B \rightarrow N}, \phi^{N \rightarrow B}, \log \left(\phi^{B \rightarrow N} / \phi^{N \rightarrow B}\right), \Delta G_{B N}$ and $\Delta G_{\text {bind }}$ de depend on the concentration of ligand. 


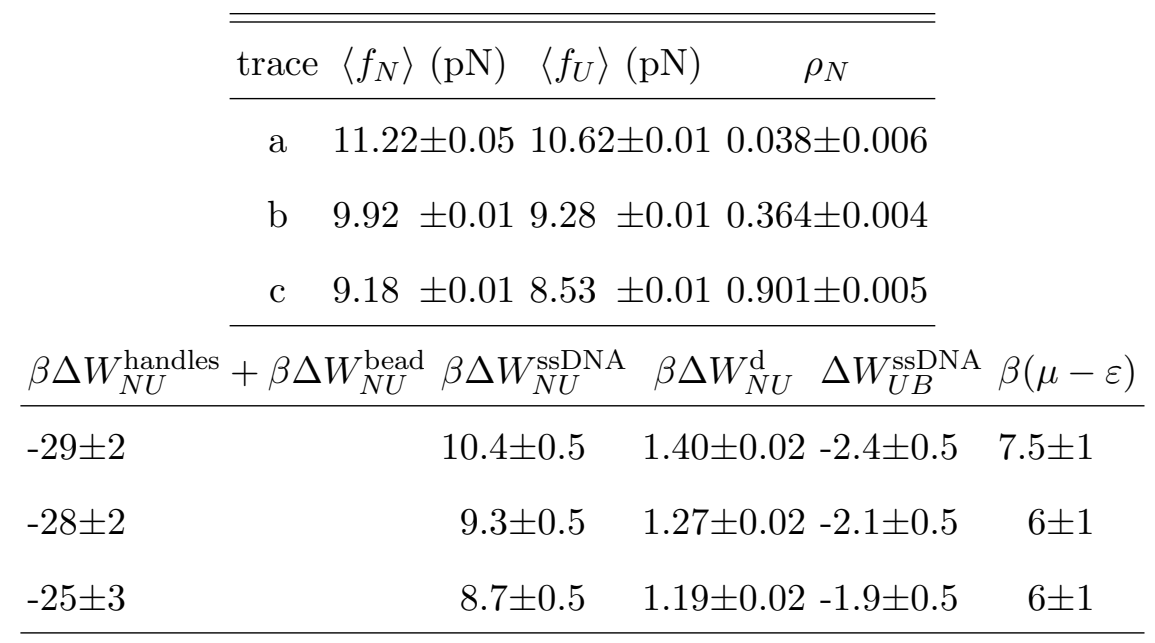

Table VII. Determination of $\beta(\mu-\varepsilon)$ in equilibrium experiments. Results obtained at $400 \mathrm{nM}$ [oligo]. 


\begin{tabular}{lll}
\hline \hline [Echi] $(\mathrm{nM})$ & pulled molecules (number of cycles per molecule) & total cycles $N$ \\
\hline 100 & $7(121,408,369,277,98,484,261)$ & 2018 \\
300 & $6(496,271,201,341,600,511)$ & 2420 \\
1000 & $9(1302,1272,486,978,420,121,327,470,196)$ & 5572 \\
3000 & $6(670,175,1010,399,1268,475)$ & 3997 \\
\hline
\end{tabular}

Table VIII. Number of experiments performed at different concentrations of echinomycin with hairpin SP. 


\begin{tabular}{|c|c|c|c|c|c|c|}
\hline$f_{B}(\mathrm{pN})$ & $f_{U}(\mathrm{pN})$ & $\Delta W_{n U}^{\mathrm{hal}}$ & $I^{\text {andles }}+\Delta W_{n U}^{\text {bead }}$ & $\Delta W$ & $\begin{array}{l}\text { ssDNA } \\
n U\end{array}$ & $\Delta W_{n U}^{d}$ \\
\hline \multicolumn{3}{|c|}{$16.77 \pm 0.02 \quad 16.25 \pm 0.02 \quad 10.5 \pm 0.3$} & $-33.0 \pm 0.8$ & \multicolumn{3}{|c|}{$8.25 \pm 0.03-1.790 \pm 0.00$} \\
\hline [Echi] $(\mathrm{nM})$ & $\phi^{B \rightarrow U}$ & $\phi^{U \rightarrow B}$ & $\log \left(\phi^{B \rightarrow U} / \phi^{U}\right.$ & $\rightarrow B)$ & $\Delta G_{B U}$ & $\Delta G_{\text {bind }}$ \\
\hline 100 & $0.5 \pm 0.1$ & $0.046 \pm 0.005$ & $-2.2 \pm 0.4$ & & $-10.8 \pm 0.4$ & $3 \pm 2$ \\
\hline 300 & $0.46 \pm 0.07$ & $0.112 \pm 0.008$ & $-1.3 \pm 0.2$ & & $-10.6 \pm 0.6$ & $5 \pm 2$ \\
\hline 1000 & $0.27 \pm 0.04$ & $0.18 \pm 0.05$ & $0.5 \pm 0.4$ & & $-10.3 \pm 0.8$ & $5 \pm 2$ \\
\hline 3000 & $0.158 \pm 0.008$ & $0.59 \pm 0.04$ & $1.3 \pm 0.1$ & & $-8.6 \pm 0.6$ & $10 \pm 1$ \\
\hline
\end{tabular}

Table IX. Contributions to the binding free energy of echinomycin to dsDNA as a function of ligand concentration [Echi]. Caption as in Table II. Here $n=1$. 


\begin{tabular}{lll}
\hline \hline [Echi] $(\mathrm{nM})$ & pulled molecules (number of cycles per molecule) & total cycles $N$ \\
\hline 100 & $6(131,145,761,492,230,567)$ & 2326 \\
300 & $8(504,506,528,455,46,320,219,275)$ & 2853 \\
1000 & $8(779,560,547,814,666,342,377,519)$ & 4604 \\
3000 & $7(310,150,268,872,241,584,533)$ & 2958 \\
\hline
\end{tabular}

Table X. Number of experiments performed at different concentrations of echinomycin with hairpin NSP. 


\begin{tabular}{|c|c|c|c|c|c|c|}
\hline$i$ & $f_{B_{i}}(\mathrm{pN})$ & & $(\mathrm{pN})$ & $\Delta G_{n_{i} U}^{0}$ & $\Delta W_{n_{i} U}^{\text {handles }}+\Delta W_{n_{i} U}^{\text {bead }}$ & $\Delta W_{n_{i} U}^{\mathrm{ssDNA}}$ \\
\hline 0 & $10.73 \pm 0.06$ & \multicolumn{2}{|c|}{$10.17 \pm 0.05$} & $19.9 \pm 0.2$ & $-26.5 \pm 0.4$ & $8.20 \pm 0.04-1.345 \pm 0.006$ \\
\hline 1 & $10.69 \pm 0.06$ & \multicolumn{2}{|c|}{$10.17 \pm 0.05$} & $16.8 \pm 0.2$ & $-24.3 \pm 0.3$ & $7.59 \pm 0.04-1.340 \pm 0.006$ \\
\hline 2 & $10.64 \pm 0.06$ & \multicolumn{2}{|c|}{$10.17 \pm 0.05$} & $13.7 \pm 0.2$ & $-22.1 \pm 0.3$ & $6.90 \pm 0.03-1.336 \pm 0.006$ \\
\hline 3 & $10.60 \pm 0.06$ & \multicolumn{2}{|c|}{$10.17 \pm 0.05$} & $11.64 \pm 0.07$ & $-20.0 \pm 0.3$ & $6.39 \pm 0.03-1.332 \pm 0.006$ \\
\hline 4 & $10.55 \pm 0.06$ & \multicolumn{2}{|c|}{$10.17 \pm 0.05$} & $10.5 \pm 0.1$ & $-17.8 \pm 0.3$ & $5.79 \pm 0.03-1.327 \pm 0.006$ \\
\hline 5 & $10.50 \pm 0.06$ & \multicolumn{2}{|c|}{$10.17 \pm 0.05$} & $8.43 \pm 0.02$ & $-15.6 \pm 0.4$ & $5.19 \pm 0.02-1.323 \pm 0.006$ \\
\hline 6 & $10.48 \pm 0.06$ & \multicolumn{2}{|c|}{$10.17 \pm 0.05$} & $4.63 \pm 0.06$ & $-13.8 \pm 0.5$ & $4.61 \pm 0.02-1.320 \pm 0.006$ \\
\hline 7 & $10.43 \pm 0.06$ & \multicolumn{2}{|c|}{$10.17 \pm 0.05$} & $2.5 \pm 0.1$ & $-11.6 \pm 0.5$ & $4.02 \pm 0.02-1.316 \pm 0.006$ \\
\hline 8 & $10.38 \pm 0.06$ & \multicolumn{2}{|c|}{$10.17 \pm 0.05$} & $1.4 \pm 0.1$ & $-9.4 \pm 0.5$ & $3.44 \pm 0.02-1.311 \pm 0.006$ \\
\hline 9 & $10.33 \pm 0.06$ & \multicolumn{3}{|c|}{$10.17 \pm 0.05-0.11 \pm 0.06$} & $-7.2 \pm 0.6$ & $2.87 \pm 0.01-1.307 \pm 0.006$ \\
\hline 10 & \multirow{2}{*}{$\begin{array}{l}10.29 \pm 0.06 \\
10.24 \pm 0.06\end{array}$} & \multicolumn{3}{|c|}{$10.17 \pm 0.05-1.18 \pm 0.03$} & $-5.1 \pm 0.6$ & $2.29 \pm 0.01-1.302 \pm 0.006$ \\
\hline 11 & & 10.1 & $7 \pm 0.05$ & $-2.71 \pm 0.03$ & $-2.9 \pm 0.7$ & $1.73 \pm 0.01-1.296 \pm 0.006$ \\
\hline & \multicolumn{2}{|c|}{ [Echi] $(\mathrm{nM})$} & $\phi^{B \rightarrow t}$ & $\phi^{U \rightarrow B}$ & $\log \left(\phi^{B \rightarrow U} / \phi^{U \rightarrow B}\right)$ & $\Delta G_{B U} \quad \Delta G_{\text {bind }}$ \\
\hline & 100 & & $0.70 \pm 0$ & $.070 .12 \pm 0.03$ & $1.9 \pm 0.2$ & $-4.5 \pm 0.2 \quad-2 \pm 1$ \\
\hline & 300 & & $0.53 \pm 0$ & $.070 .30 \pm 0.01$ & $0.5 \pm 0.1$ & $-3.3 \pm 0.2$ \\
\hline & 1000 & & $0.37 \pm 0$ & $.020 .46 \pm 0.03$ & $-0.2 \pm 0.1$ & $-2.6 \pm 0.2$ \\
\hline & 300 & & $0.21 \pm 0$ & $.040 .70 \pm 0.05$ & $-1.3 \pm 0.3$ & $-1.2 \pm 0.4$ \\
\hline
\end{tabular}

Table XI. Contributions to the binding free energy of echinomycin to dsDNA as a function of ligand concentration [Echi]. The term $\mathcal{N}$ is taken equal to $\mathcal{N}=12$, which is equal to the number of basepairs of H.NSP. The term $\log \sum_{i} \exp \left(-\beta \Delta G_{n_{i} U}\right)$ is equal to $7.6 \pm 0.5 k_{\mathrm{B}} T$. 
Hairpin pulled molecules (number of cycles per molecule) total cycles $N$

HC $\quad 7(40,348,312,324,603,352,374) \quad 2353$

HNC $6(997,300,193,325,845,125) \quad 2785$

Table XII. Number of experiments performed at $3000 \mathrm{nM}$ [Echi] with hairpins C and NC. 


\begin{tabular}{|c|c|c|c|c|}
\hline Hairpin & $f_{B^{2}}(\mathrm{pN})$ & $f_{U}(\mathrm{pN})$ & $\Delta W_{n_{2} U}^{\text {handles }}+\Delta W_{n_{2} U}^{\text {bead }}$ & $\Delta W_{n_{2} U}^{\mathrm{ssDNA}}$ \\
\hline $\mathrm{C}$ & $20.0 \pm 0.2$ & $19.4 \pm 0.2 \quad 18.3 \pm 0.8$ & $-51.1 \pm 0.4$ & $12.10 \pm 0.07-1.967 \pm 0.008$ \\
\hline \multirow[t]{4}{*}{$\mathrm{NC}$} & $16.67 \pm 0.05$ & $15.94 \pm 0.05 \quad 18.3 \pm 0.8$ & $-41 \pm 1$ & $10.56 \pm 0.03-1.785 \pm 0.003$ \\
\hline & Hairpin & $\phi^{U \rightarrow B^{2}}$ & $\log \left(\phi^{B^{2} \rightarrow U} / \phi^{U \rightarrow B^{2}}\right)$ & $\Delta G_{B^{2} U} \Delta G_{\text {bind }}$ \\
\hline & $\mathrm{C}$ & $0.26 \pm 0.05 \quad 0.72 \pm 0.03$ & $-1.1 \pm 0.3$ & $-14 \pm 1 \quad 4.5 \pm 0.5$ \\
\hline & $\mathrm{NC}$ & $0.15 \pm 0.02$ & $0.9 \pm 0.3$ & $-8 \pm 1 \quad 2.6 \pm 0.4$ \\
\hline
\end{tabular}

Table XIII. Contributions to the binding free energy for double binding to dsDNA at $3000 \mathrm{nM}$ echinomycin. At $3000 \mathrm{nM}$ [Echi] double binding events of echinomycin to hairpins $\mathrm{C}$ and NC are always observed. $n_{2}=4$ is the number of open basepairs when two echinomycin molecules bind to specific sites in H.C or H.NC. 


\begin{tabular}{|c|c|c|c|c|c|c|c|}
\hline Hairpin & $f_{B}(\mathrm{pN})$ & $f_{U}(\mathrm{pN})$ & $\Delta G_{n_{1} U}^{0}$ & $\Delta W_{n_{1} U}^{\text {handles }}+\Delta W$ & $V_{n_{1} U}^{\text {bead }}$ & $\Delta W_{n_{1} U}^{\mathrm{ssDNA}}$ & $\Delta W_{n_{1} U}^{d}$ \\
\hline \multirow[t]{3}{*}{$\mathrm{NC}$} & $16.1 \pm 0.2$ & $15.95 \pm 0.0$ & $6.6 \pm 0.4$ & $-15 \pm 2$ & & $5.65 \pm 0.01$ & $-1.757 \pm 0.006$ \\
\hline & $\phi^{B}$ & $\rightarrow U$ & $\rightarrow B$ & $g\left(\phi^{B \rightarrow U} / \phi^{U \rightarrow B}\right)$ & $\Delta G_{B l}$ & $3 U \Delta G_{\text {bind }}$ & \\
\hline & $0.6=$ & \pm 0.20 .031 & \pm 0.006 & $3.1 \pm 0.3$ & $-9 \pm 1$ & $-5 \pm 2$ & \\
\hline
\end{tabular}

Table XIV. Contributions to the binding free energy for single binding to dsDNA at 3000 nM echinomycin. Even though when pulling hairpin NC at $3000 \mathrm{nM}$ [Echi] all binding events correspond to double binding, single binding events are also transiently observed with hairpin NC at large forces when the echimoycin molecule bound at the start of the hairpin stem spontaneously unbinds. Forward and reverse trajectories connecting such transient single binding state and the unfolded state also provide a measurement of $\Delta G_{\text {bind }}$ for hairpin H.NC. 
pulled molecules (number of cycles per molecule) total cycles $N$

$3(101,300,314)$

715

Table XV. Number of experiments performed at $10 \mu \mathrm{M}$ [Echi] with hairpins $\mathbf{M}$. 


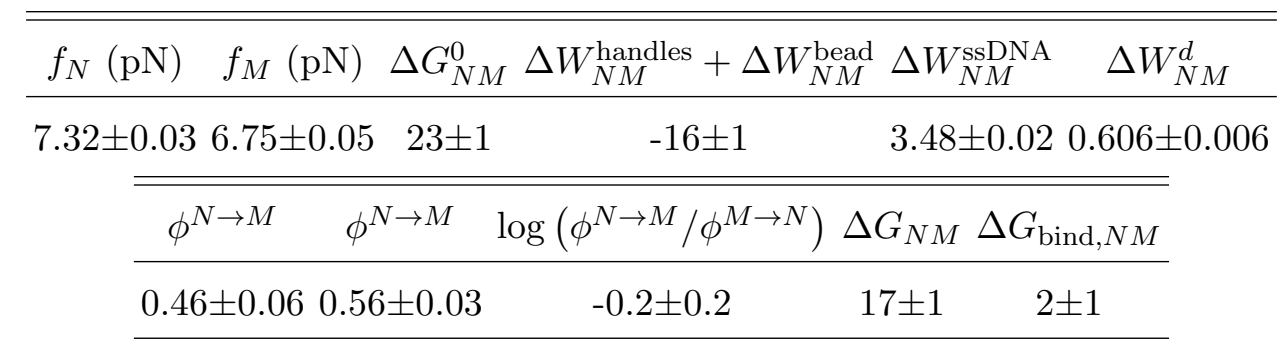

Table XVI. Double binding of two echinomycin molecules to hairpin M stabilizes a mis-

folded state. In the simultaneous binding of two echinomycin molecules to the folded hairpin $\mathrm{M}$ two unrelated structures are observed: one corresponds to the native state whereas the other correspond to a misfolded structure (Fig. S1 and 10). The difference in binding energy of echinomycin to each of the two structures is $\Delta G_{\text {bind,NM }}=2 \pm 1 k_{\mathrm{B}} T$ according to our results. 
[1] G. D. Stormo, \& Y. Zhao. Determining the specificity of protein-DNA interactions. Nat. Rev. Genet. 11, 751-760 (2010).

[2] S. Leavitt, \& E. Freire. Direct measurement of protein binding energetics by isothermal titration calorimetry. Curr. Opin. Struct. Biol. 11, 560-566 (2001).

[3] J. M. McDonnell. Surface plasmon resonance: towards an understanding of the mechanisms of biological molecular recognition. Curr. Opin. Chem. Biol. 5, 572-577 (2001).

[4] C. G. Kalodimos, et al. Structure and flexibility adaptation in nonspecific and specific proteinDNA complexes. Science 305, 386-389 (2004).

[5] S. Kim, et al. Probing allostery through DNA. Science 339, 816-819 (2013).

[6] C. Bustamante, Z. Bryant, \& S.B. Smith. Ten years of tension: single-molecule DNA mechanics. Nature 421, 423-427 (2003).

[7] J.P. Junker, K. Hell, M. Schlierf, W. Neupert, \& M. Rief. Influence of substrate binding on the mechanical stability of mouse dihydrofolate reductase. Biophys. J. 89, L46-L48 (2005).

[8] Y. Cao, M.M. Balamurali, D. Sharma, \& H. Li. A functional single-molecule binding assay via force spectroscopy. Proc. Natl. Acad. Sci. U.S.A. 104, 15677-15681 (2007).

[9] D. Koirala, et al. A single-molecule platform for investigation of interactions between Gquadruplexes and small-molecule ligands. Nature Chem. 3, 782-787 (2011).

[10] S. R. K. Ainavarapu, L. Lewyn, C. Badilla, \& J.M. Fernandez Ligand binding modulates the mechanical stability of dihydrofolate reductase Biophys. J. 89, 3337-44 (2005).

[11] H. Eleanore, et al. The effect of protein complexation on the mechanical stability of Im9. Biophys. J. 92, L79-L81 (2007).

[12] C. Jarzynski. Nonequilibrium equality for free energy differences. Phys. Rev. Lett. 78, 2690 (1997).

[13] G. E. Crooks. Path-ensemble averages in systems driven far from equilibrium. Phys. Rev. E 61, 2361 (2000).

[14] J. Liphardt, S. Dumont, S. B. Smith, I. Tinoco, \& C. Bustamante. Equilibrium information from nonequilibrium measurements in an experimental test of Jarzynski's equality. Science 296, 1832-1835 (2002).

[15] D. Collin, et al. Verification of the crooks fluctuation theorem and recovery of RNA folding 
free energies. Nature 437, 231-234 (2005).

[16] E. A. Shank, C. Cecconi, J. W. Dill, S. Marqusee, \& C. Bustamante. The folding cooperativity of a protein is controlled by its chain topology. Nature 465, 637-640 (2010).

[17] P. Maragakis, M. Spichty, \& M. Karplus. A differential fluctuation theorem. J. Phys. Chem. $B$ 112, 6168-6174 (2008).

[18] I. Junier, A. Mossa, M. Manosas, \& F. Ritort. Recovery of free energy branches in single molecule experiments. Phys. Rev. Lett. 102, 070602 (2009).

[19] A. Alemany, A. Mossa, I. Junier, \& F. Ritort. Experimental free-energy measurements of kinetic molecular states using fluctuation theorems. Nature Phys. 8, 688-694 (2012).

[20] Materials and methods are available as supplementary materials on Science Online.

[21] A. Orte, et al. Direct characterization of amyloidogenic oligomers by single-molecule fluorescence. Proc. Natl. Acad. Sci. U.S.A. 105, 14424-14429 (2008).

[22] B. Fierz, S. Kilic, A. R. Hieb, K. Luger, \& T. W. Muir. Stability of nucleosomes containing homogenously ubiquitylated H2A and H2B prepared using semisynthesis. J. Am. Chem. Soc. 134, 19548-19551 (2012).

[23] H. Yu, et al. Direct observation of multiple misfolding pathways in a single prion protein molecule. Proc. Natl. Acad. Sci. U.S.A. 109, 5283-5288 (2012).

[24] D. R. Lesser, M. R. Kurpiewski, \& L. Jen-Jacobson. The energetic basis of specificity in the EcoRI endonuclease-DNA interaction. Science 250, 776-786 (1990).

[25] L. Jen-Jacobson. Protein-DNA recognition complexes: Conservation of structure and binding energy in the transition state. Biopolymers 44, 153-180 (1997).

[26] B. Terry, W. Jack, R. Rubin, \& P. Modrich. Thermodynamic parameters governing interaction of EcoRI endonuclease with specific and nonspecific DNA sequences. J. Biol. Chem. 258, 9820-9825 (1983).

[27] S. J. Koch, A. Shundrovsky, B. C. Jantzen, \& M. D. Wang. Probing protein-DNA interactions by unzipping a single DNA double helix. Biophys. J. 83, 1098-1105 (2002).

[28] J. SantaLucia. A unified view of polymer, dumbbell, and oligonucleotide DNA nearestneighbor thermodynamics. Proc. Natl. Acad. Sci. U.S.A. 95, 1460-1465 (1998).

[29] J. M. Huguet, et al. Single-molecule derivation of salt dependent base-pair free energies in DNA. Proc. Natl. Acad. Sci. U.S.A. 107, 15431-15436 (2010).

[30] M. M. Van Dyke, \& P. B. Dervan. Echinomycin binding sites on DNA. Science 225, 1122-1127 
(1984).

[31] C. Bailly, F. Hamy, \& M. J. Waring. Cooperativity in the binding of echinomycin to DNA fragments containing closely spaced CpG sites. Biochemistry 35, 1150-1161 (1996).

[32] F. Leng, J. B. Chaires, \& M. J. Waring. Energetics of echinomycin binding to DNA. Nucleic Acids Res. 31, 6191-6197 (2003).

[33] P. O. Heidarsson, et al. Direct single-molecule observation of calcium-dependent misfolding in human neuronal calcium sensor-1. Proc. Natl. Acad. Sci. U.S.A. 111, 13069-13074 (2014).

[34] A. Thåström, J. Gottesfeld, K. Luger, \& J. Widom. Histone-DNA binding free energy cannot be measured in dilution-driven dissociation experiments. Biochemistry 43, 736-741 (2004).

[35] S. Schnell, \& T. E. Turner. Reaction kinetics in intracellular environments with macromolecular crowding: simulations and rate laws. Prog. Biophys. Mol. Bio. 85, 235-260 (2004).

[36] N. Forns, et al. Improving signal/noise resolution in single-molecule experiments using molecular constructs with short handles. Biophys. J. 100, 1765-1774 (2011).

[37] W. Cheng, S. G. Arunajadai, J. R. Moffitt, I. Tinoco, \& C. Bustamante. Single base pair unwinding and asynchronous RNA release by the hepatitis C virus NS3 helicase. Science 333, 1746-1749 (2011).

[38] N. Y. Sidorova, \& D. C. Rau. Differences in water release for the binding of EcoRI to specific and nonspecific DNA sequences. Proc. Natl. Acad. Sci. U.S.A. 93, 12272-12277 (1996).

[39] M. Zuker. Mfold web server for nucleic acid folding and hybridization prediction. Nucleic Acids Res. 31, 3406-3415 (2003).

[40] C. H. Bennett. Efficient estimation of free energy differences from Monte Carlo data. J. Comput. Phys. 22, 245-268 (1976).

[41] M. R. Shirts, E. Bair, G. Hooker, \& V. S. Pande. Equilibrium free energies from nonequilibrium measurements using maximum likelihood methods. Phys. Rev. Lett. 91, 140601 (2003).

[42] M. J. Comstock, T. Ha, \& Y. R. Chemla. Ultrahigh-resolution optical trap with singlefluorophore sensitivity. Nat. Methods 8, 335-340 (2011).

[43] J. Camunas-Soler, et al. Single-molecule kinetics and footprinting of DNA bis-intercalation: the paradigmatic case of Thiocoraline. Nucleic Acids Res. 43, 2767-2779 (2015).

[44] A. Alemany, \& F. Ritort. Determination of the elastic properties of short ssDNA molecules by mechanically folding and unfolding DNA hairpins. Biopolymers 101, 1193-1199 (2014). 